\title{
The Integration of Mathematics Discourse, Graphical Reasoning and Symbolic Expression by a Virtual Math Team
}

\author{
Murat Perit Çakır, Informatics Institute, Middle East Technical University, Ankara, Turkey \\ Gerry Stahl, School of Information Science \& Technology, Drexel University, Philadelphia, USA
}

\begin{abstract}
Learning mathematics involves mastering specific forms of social practice. In this chapter, we describe socially situated, interactional processes involved with collaborative learning of mathematics online. We provide a group-cognitive account of mathematical understanding in an empirical case study of an online collaborative learning environment called Virtual Math Teams. The chapter looks closely at how an online small group of mathematics students coordinates their collaborative problem solving using chat, shared drawings and mathematics symbols. Our analysis highlights the methodic ways group members enact the affordances of their situation (a) to display their reasoning to each other by co-constructing shared mathematical artifacts and (b) to coordinate their actions across multiple interaction spaces to relate their narrative, graphical and symbolic contributions while they are working on open-ended mathematics problems. In particular, we identify key roles of referential and representational practices in the co-construction of deep mathematical understanding at the group level, which is achieved through methodic uses of the environment's features to coordinate narrative, graphical and symbolic resources.
\end{abstract}

\section{Mathematical Practices}

Developing pedagogies and instructional tools to support learning mathematics with understanding is a major goal in Mathematics Education (CCSSI, 2011; NCTM, 2000). A common theme among various characterizations of mathematical understanding in the mathematics education literature involves constructing relationships among mathematical facts and procedures (Hiebert \& Wearne, 1996). In particular, recognition of connections among multiple realizations of a mathematics concept encapsulated in various inscriptional forms is considered as evidence of deep understanding of that subject matter (Kaput, 1998; Sfard, 2008; Healy \& Hoyles, 1999). For instance, the concept of function in the modern mathematics curriculum is introduced through its graphical, narrative, tabular and symbolic realizations. Hence, a deep understanding of the function concept is ascribed to a learner to the extent he/she can demonstrate how seemingly different graphical, narrative and symbolic forms are interrelated as realizations of each other in specific problem-solving circumstances that require the use of functions. On the other hand, students who demonstrate difficulties in realizing such connections are considered to perceive actions associated with distinct forms as isolated sets of skills, and hence are said to have a shallow understanding of the subject matter (Carpenter \& Lehrer, 1999).

Reflecting on one's own actions and communicating/articulating mathematical rationale are considered as important activities through which students realize connections among seemingly isolated facts and procedures in mathematics education theory (Sfard, 2002; Hiebert et al., 1996). Such activities are claimed to help learners notice broader structural links among underlying concepts, reorganize their thoughts around these structures, and hence develop their understanding of mathematics (Carpenter \& Lehrer, 1999; Skemp, 1976). Consequently, collaborative learning in peer-group settings is receiving increasing interest in mathematics education practice due to its potential for promoting student participation and creating a natural setting where students can explain their reasoning and benefit from each others' perspectives (Barron, 2003).

Representational capabilities offered by Information and Communication Technologies (ICT) provide important affordances for exploring connections among different realizations of mathematical objects. 
Dynamic geometry applications like Cabri, Geometer's Sketchpad, GeoGebra (Goldenberg \& Cuoco, 1998); algebra applications such as Casyospee (Lagrange, 2005), or statistical modeling and exploratory data analysis tools like TinkerPlots (Konold, 2007) provide representational capabilities and virtual manipulatives that surpass what can be done with conventional methods of producing mathematical inscriptions in the classroom (Olive, 1998). In addition to this, widespread popularity of social networking and instant messaging technologies among the so-called Net Generation requires designers of educational technology to think about innovative ways for engaging the new generation of students with mathematical activity (Lenhart et al., 2007). Therefore, bringing the representational capabilities of existing mathematical packages together with communicational affordances of socialnetworking/messenger software can potentially support the kinds of interactions that foster deeper understanding of mathematics. Computer-Supported Collaborative Learning (CSCL) is a research paradigm in the field of Instructional Technology that investigates how such opportunities can be realized through carefully designed learning environments that support collective meaning-making practices in computer-mediated settings (Stahl, Koschmann, \& Suthers, 2006).

Multimodal interaction spaces - which typically bring together two or more synchronous online communication technologies such as text-chat and a shared graphical workspace- have been widely employed in CSCL research and in commercial collaboration suites such as Elluminate and BlackboardWimba to support collaborative-learning activities of small groups online (Dillenbourg \& Traum, 2006; Suthers et al., 2001). The way such systems are designed as a juxtaposition of several technologically independent online communication tools not only brings various affordances (i.e., possibilities for and/or constraints on actions), but also carries important interactional consequences for the users (Cakir, Zemel \& Stahl, 2009; Suthers, 2006; Dohn 2009). Providing access to a rich set of modalities for action allows users to demonstrate their reasoning in multiple semiotic forms. However, the achievement of connections that foster the kind of mathematical understanding desired by mathematics educators is conditioned upon team members' success in devising shared methods for coordinated use of these resources (Mühlpfordt \& Stahl, 2007).

Although CSCL environments with multimodal interaction spaces offer rich possibilities for the creation, manipulation, and sharing of mathematical artifacts online, the interactional organization of mathematical meaning-making activities in such online environments is a relatively unexplored area in CSCL and in mathematics education. In an effort to address this gap, we have designed an online environment with multiple interaction spaces called Virtual Math Teams (VMT), which allows users to exchange textual postings as well as share graphical contributions online (Stahl, 2009). The VMT environment also provides additional resources, such as explicit referencing and special awareness markers, to help users coordinate their actions across multiple spaces. Of special interest to researchers, this environment includes a Replayer tool to replay a chat session as it unfolded in real time and inspect how students organize their joint activity to achieve the kinds of connections indicative of deep understanding of mathematics (Stahl, 2011).

In this chapter we focus on the interactional practices through which VMT participants achieve the kinds of connections across multiple semiotic modalities that are indicative of deep mathematical understanding. In particular, the chapter will look closely at how an online small group of mathematics students coordinated their collaborative problem solving using digital text, drawings and symbols. We take the mathematics-education practitioners' account of what constitutes deep learning of mathematics as a starting point, but instead of treating understanding as a mental state of the individual learner that is typically inferred by outcome measures, we argue that deep mathematical understanding can be located in the practices of collective multimodal reasoning displayed by groups of students through the sequential and spatial organization of their actions (Stahl, 2006). In an effort to study the practices of multimodal reasoning online, we employ an ethnomethodological case-study approach and investigate the methods through which small groups of students achieve joint attention to particular mathematical features of their 
representations in order to ground their co-construction of shared mathematical meaning (Sarmiento \& Stahl, 2008, Stahl, et al., 2011). Our analysis of the excerpts presented below has identified key roles of referential and representational practices in the co-construction of deep mathematical understanding at the group level, which is elaborated further in the discussion section.

\section{Data Collection \& Methodology}

The excerpts analyzed in this chapter are obtained from a problem-solving session of a team of three upper-middle-school students who participated in the VMT Spring Fest 2006. This event brought together several teams from the US, Singapore and Scotland to collaborate on an open-ended mathematics task on combinatorial patterns. Students were recruited anonymously through their teachers. Members of the teams generally did not know each other before the first session. Neither they nor we knew anything about each other (e.g., age or gender) except chat screen names and information that may have been communicated during the sessions. Each group participated in four sessions during a two-week period, and each session lasted over an hour. Each session was moderated by a Math Forum member; the facilitators' task was to help the teams when they experienced technical difficulties, not to participate in the problem-solving work.

During their first session, all the teams were asked to work on a particular pattern of squares made up of sticks (see Figure 1). For the remaining three sessions the teams were asked to come up with their own stick patterns, describe the patterns they observed as mathematical formulae, and share their observations with other teams through a wiki page.

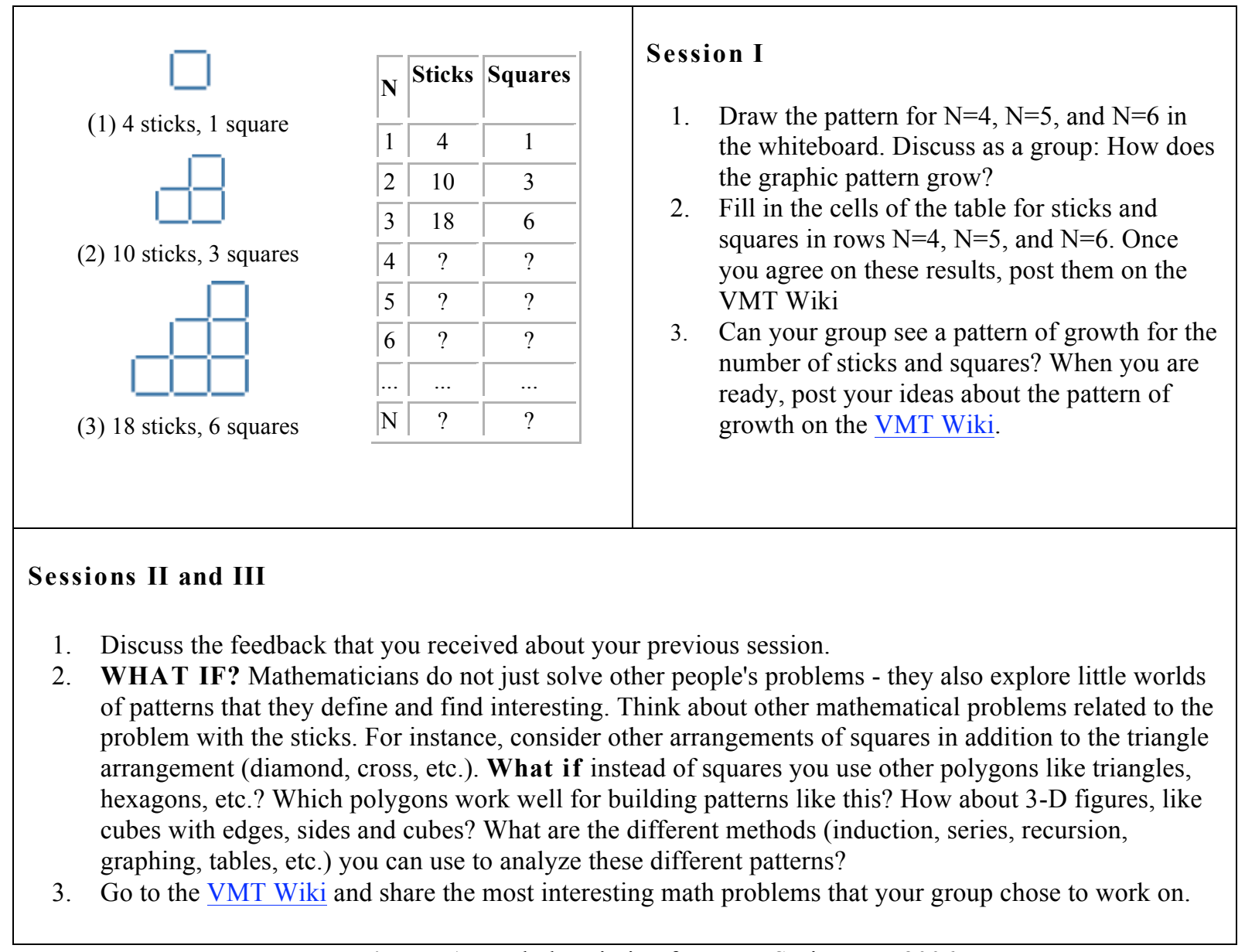

Figure 1: Task description for VMT Spring Fest 2006 
This task was chosen because of the possibilities it afforded for many different solution approaches ranging from simple counting procedures to more advanced methods, such as the use of recursive functions and exploring the arithmetic properties of various number sequences. Moreover, the task had both algebraic and geometric aspects, which would potentially allow us to observe how participants put many features of the VMT software system into use. The open-ended nature of the activity stemmed from the need to agree upon a new shape made by sticks. This required groups to engage in a different kind of problem-solving activity as compared to traditional situations where questions are given in advance and there is a single "correct" answer-presumably already known by a teacher. We used a traditional problem to seed the activity and then left it up to each group to decide the kinds of shapes they found interesting and worth exploring further (Moss \& Beatty, 2006; Watson \& Mason, 2005).

The VMT system that hosted these sessions has two main interactive components that conform to the typical layout of systems with dual-interaction spaces: a shared whiteboard that provides basic drawing features on the left and a chat window on the right. The online environment has features to help users relate the actions happening across dual-interaction spaces. One of the unique features of the VMT chat system is the referencing support mechanism (Mühlpfordt \& Stahl, 2007), which allows users to visually connect their chat postings to previous postings or to objects on the whiteboard via arrows (e.g., Figure 7 below illustrates a message-to-message reference, whereas Figure 6 shows a message-to-whiteboard reference). The referential links attached to a message are displayed until a new message is posted. Messages including referential links are marked with an arrow icon in the chat window. A user can see where such a message is pointing at any time by clicking on it.

Studying the collective meaning-making practices enacted by the users of CSCL systems requires a close analysis of the process of collaboration itself (Stahl, Koschmann \& Suthers, 2006; Koschmann, Stahl \& Zemel, 2007). In an effort to investigate the organization of interactions across the dual-interaction spaces of the VMT environment, we consider the small group as the unit of analysis (Stahl, 2006), and we appropriate methods of Ethnomethodology (EM) (Garfinkel, 1967; Livingston, 1987) and Conversation Analysis (CA) (Sacks, 1962/1995; ten Have, 1999) to conduct case studies of online group interaction. Our work is informed by EM/CA studies of interaction mediated by online text-chat (Garcia \& Jacobs, 1998; O'Neill \& Martin, 2003), although the availability of a shared drawing area and explicit support for deictic references in our online environment, as well as our focus on mathematical practice significantly differentiate our study from theirs.

The goal of ethnomethodological conversation analysis is to describe the commonsense understandings and procedures group members use to organize their conduct in particular interactional settings. Commonsense understandings and procedures are subjected to analytical scrutiny because they "enable actors to recognize and act on their real world circumstances, grasp the intentions and motivations of others, and achieve mutual understandings" (Goodwin \& Heritage, 1990, p. 285). Group members' shared competencies in organizing their conduct not only allow them to produce their own actions, but also to interpret the actions of others (Garfinkel \& Sacks, 1970). Since members enact these understandings and/or procedures in their visually displayed situated actions, researchers can discover them through detailed analysis of members' sequentially organized conduct (Schegloff \& Sacks, 1973).

We conducted numerous VMT Project data sessions, where we subjected our analysis of VMT data to intersubjective agreement by conducting CA data sessions (Jordan \& Henderson, 1995; ten Have, 1999). During the data sessions we used the VMT Replayer tool, which allows us to replay a VMT chat session as it unfolded in real time based on the timestamps of actions recorded in the log file. The order of actions - chat postings, whiteboard actions, awareness messages - we observe with the Replayer as researchers exactly matches the order of actions originally observed by the users. This property of the Replayer allowed us to study the sequential unfolding of events during the entire chat session. In short, 
the VMT environment provided us as researchers a perspicuous setting in which the mathematical meaning-making process is made visible as it is "observably and accountably embedded in collaborative activity" (Koschmann, 2001, p. 19).

\section{Setting Up the Mathematical Analysis}

In the following excerpts we will observe a team of three upper-middle-school students in action, who used "Qwertyuiop", "137" and "Jason" as login screen names. Prior to the session containing these excerpts, this team completed two chat sessions where they explored similar stick patterns together. In the current session, team members will be working on a new stick pattern that they co-constructed and named as the "hexagonal pattern", whose first three stages are illustrated in Figure 2. Details of this coconstruction process was analyzed and published elsewhere (Cakir, Zemel \& Stahl, 2009; Cakir, 2009), so we will skip the part where the group constituted this pattern as a shared problem and figured out a method to count the number of triangles enclosed in its $\mathrm{n}^{\text {th }}$ stage. In the excerpts presented below, the team will be working on devising a formula for characterizing the number of sticks that will be needed to construct the hexagonal pattern in general (i.e., in its $n^{\text {th }}$ stage). Our main analytic goal is to identify the practices or group methods team members enacted to achieve a shared understanding of the problem at hand by co-constructing and acting on the mathematical artifacts in graphical, narrative and symbolic forms.
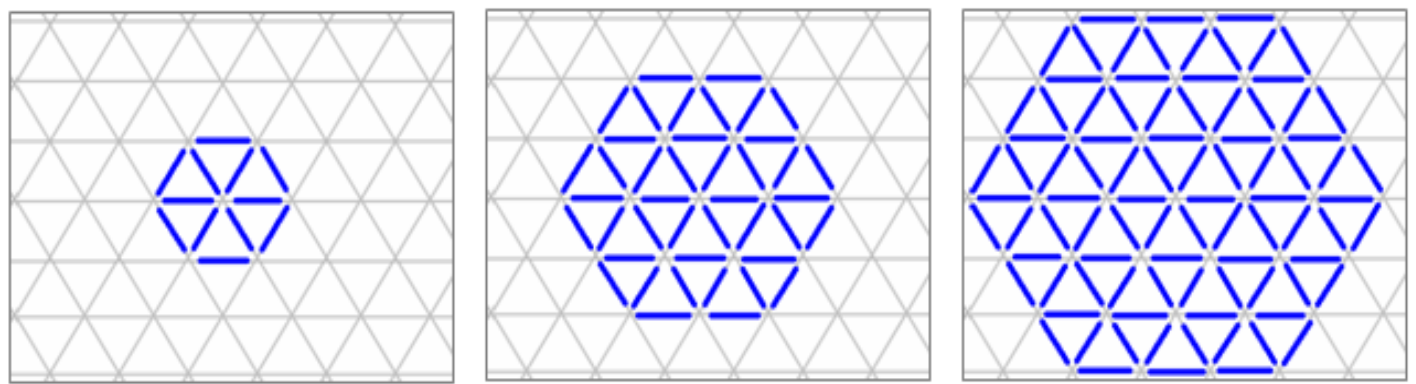

Figure 2: Hexagonal stick pattern co-constructed by this team

Excerpt 1: Constitution of a New Math Task

\begin{tabular}{|l|l|l|l|l|l|}
\hline $\begin{array}{l}\text { Chat } \\
\text { Index }\end{array}$ & $\begin{array}{l}\text { Time } \\
\text { Start } \\
\text { Typing }\end{array}$ & $\begin{array}{l}\text { Time of } \\
\text { Posting }\end{array}$ & Author & Content & Refers to \\
\hline 742 & $19: 24: 39$ & $19: 25: 48$ & Qwertyuiop & $\begin{array}{l}\text { an idea: Find the number of a certain set } \\
\text { of colinear sides (there are 3 sets) and } \\
\text { multiply the result by 3 }\end{array}$ & \\
\hline 743 & $19: 25: 55$ & $19: 26: 03$ & Jason & i did--apparently it didn't work for him & Message No. 740 \\
\hline 744 & $19: 26: 05$ & $19: 26: 13$ & Jason & $\begin{array}{l}\text { or his internet could be down, as he's not } \\
\text { even on IM right now }\end{array}$ & \\
\hline 745 & $19: 26: 10$ & $19: 26: 13$ & Nan & i see. thanks & Message No. 743 \\
\hline & & $19: 26: 23-$ & & $\begin{array}{l}\text { 137 produces two green lines on the } \\
\text { diagonals of the hexagon and two green } \\
\text { arrows as displayed in Figure 3 }\end{array}$ & \\
\hline 746 & $19: 26: 20$ & $19: 26: 36$ & 137 & As in those? & Message No. 742 \\
\hline 747 & $19: 26: 46$ & $19: 27: 05$ & Qwertyuiop & no-in one triangle. I'll draw it... & Message No. 746 \\
\hline & & $19: 27: 10-$ & & $\begin{array}{l}\text { Qwertyuiop repositions some of the } \\
\text { existing green lines } \text { on a particular } \\
\text { section of the hexagon (see Figure } 4\end{array}$ & \\
below) & \\
\hline
\end{tabular}




\begin{tabular}{|c|c|c|c|c|}
\hline 748 & 19:28:09 & 19:28:10 & Qwertyuiop & Those \\
\hline & & $\begin{array}{l}19: 28: 13- \\
19: 28: 19\end{array}$ & & $\begin{array}{l}137 \text { makes the green lines thicker (see } \\
\text { Figure } 4 \text { below) }\end{array}$ \\
\hline 749 & & $19: 28: 28$ & Qwertyuiop & find those, and then multiply by 3 \\
\hline 750 & 19:28:48 & 19:28:50 & 137 & The rows? \\
\hline 751 & 19:29:01 & 19:30:01 & Qwertyuiop & $\begin{array}{l}\text { The green lines are all colinear. There are } \\
3 \text { identical sets of colinear lines in that } \\
\text { triangle. Find the number of sides in one } \\
\text { set, then multiply by } 3 \text { for all the other } \\
\text { sets. }\end{array}$ \\
\hline 752 & $19: 30: 20$ & $19: 30: 23$ & 137 & Ah. I see. \\
\hline
\end{tabular}

This excerpt illustrates a number of rich referencing methods: special terms, graphical practices, VMT tools, etc. Excerpt $1^{1}$ opens with Qwertyuiop's announcement of "an idea"' in line 742. He suggests the team find the number of a set of objects he calls "collinear sides" and multiply that number by 3 . The statement in parenthesis elaborates further that there are 3 such sets. The use of the term "sides" makes it evident that this statement is about the problem of finding the number of sticks to construct a given stage, rather than the problem of finding the number of triangles that make up a hexagon that has been recently discussed by the team ${ }^{3}$. Thus, Qwertyuiop seems to be proposing to his teammates a way to approach the problem of counting the number of sticks needed to construct the hexagonal shape in general.

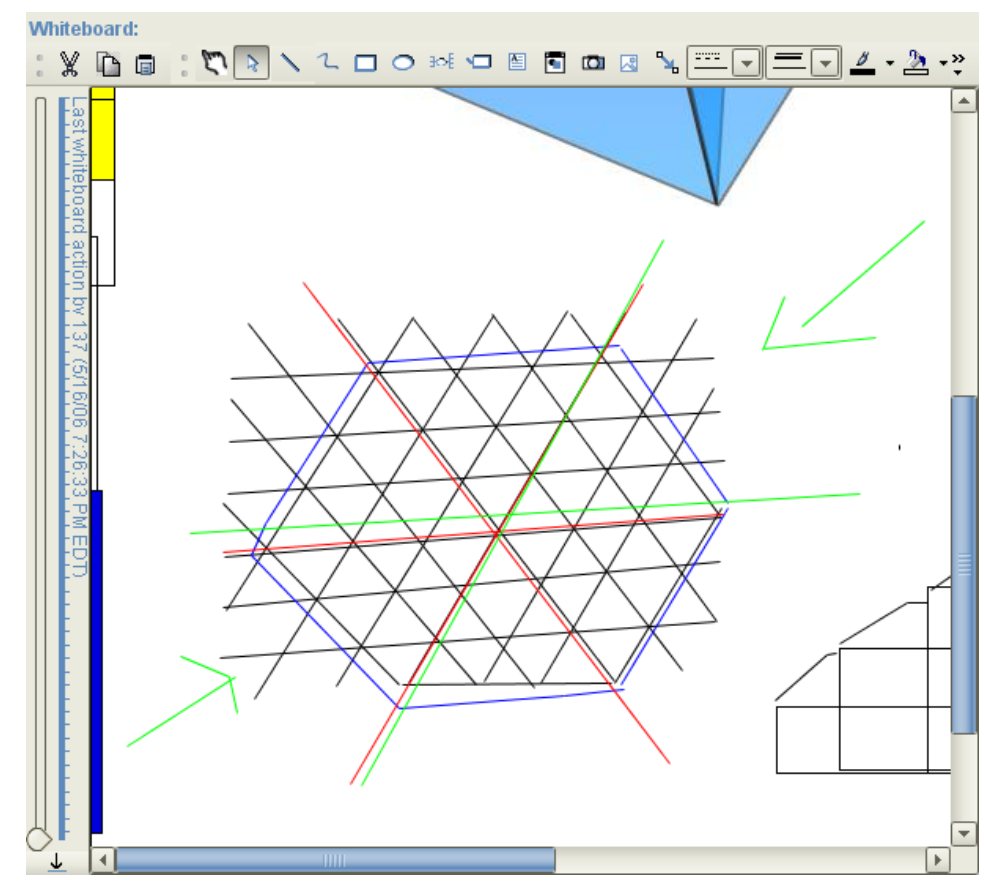

Figure 3: Green lines and arrows produced by 137.

\footnotetext{
1 The referential links used by the students to connect their messages to previous messages are displayed in the right-most column in the excerpts. For instance, line 745 includes Message \#742 in the right-most column. This indicates that message 745 was linked to 742 by its contributor (i.e. Nan in this case). References to whiteboard objects are also marked in this column. Whiteboard drawing actions are described in bold-italics to separate them from chat messages. Note that chat postings and whiteboard drawings often interleave each other.

${ }^{2}$ Phrases quoted from chat messages are printed in bold to highlight the terms used by the participants.

${ }^{3}$ There is a parallel conversation unfolding in chat at this moment between the facilitator (Nan) and Jason about an administrative matter. Lines 740, 743, 744, and 745 are omitted from the analysis to keep the focus on the math problem solving.
} 
A minute after this posting, 137 begins to type at 19:26:20. While the awareness marker continues to display that 137 is typing, he adds two green lines on the hexagon that intersect each other and two green arrows (see Figure 3). The arrows are positioned outside the hexagon and their tips are mutually pointing at each other through a projected diagonal axis. Shortly after his last drawing move, 137 completes his typing action by posting the message "as in those?" in line 746, which is explicitly linked to Qwertyuiop's previous posting with a referential arrow. The plural ${ }^{4}$ deictic term "those" in this posting instructs others to attend to some objects beyond the chat statement itself, possibly located in the other interaction space. The way the drawing actions are embedded as part of the typing activity suggests that they are designed to be seen as part of a single turn or exposition. Hence, the deictic term "those" can be read as a reference to the objects pointed to by the recently added green arrows ${ }^{5}$. Moreover, the use of the term "as" and the referential link together suggest that these drawings are related to Qwertyuiop's proposal in line 746. Therefore, based on the evidence listed above, 137 proposes a provisional graphical representation of what was described in narrative form by Qwertyuiop earlier and calls for an assessment of its adequacy.

In line 747 Qwertyuiop posts a message linked to 137's message with the referential arrow, which indicates that he is responding to 137's recent proposal. The use of "no" at the beginning expresses disagreement and the following phrase "in one triangle" gives further specificity to where the relevant relationship should be located. The next sentence "I will draw it..." in the same posting informs other members that he will continue his elaboration on the whiteboard. The use of ellipsis "..." also marks the incomplete status of this posting, which informs others that his subsequent drawings should be seen as related to this thread.

Following this line, Qwertyuiop begins to reposition some of the green lines that 137 drew earlier. He forms three green horizontal lines within one of the six triangular partitions (see the snapshot on the left in Figure 4). Then in line 748, he posts the deictic term "those" that can be read as a reference to the recently added lines. Immediately following Qwertyuiop's statement, 137 modifies the recently added lines by increasing their thickness (see the snapshot on the right in Figure 4). These moves make the new lines more visible. In line 749, Qwertyuiop continues his exposition by stating that what has been marked (indexed by "those") is what needs to be found and then multiplied by 3 .

\footnotetext{
${ }^{4} 137$ 's referential work involves multiple objects in this instance. Although the referencing tool of VMT can be used to highlight more than one area on the whiteboard, this possibility was not mentioned during the tutorial and hence was not available to the users. Although the explicit referencing tool of the system seemed to be inadequate to fulfill this complicated referential move, 137 achieves a similar referential display by temporally coordinating his moves across both interaction spaces and by using the plural deictic term "those" to index his recent moves.

${ }^{5}$ We have observed that students use "those" (or "that") in chat to reference items already existing in the whiteboard, but "these" (or "this") to reference items that they are about to add to the whiteboard.
} 

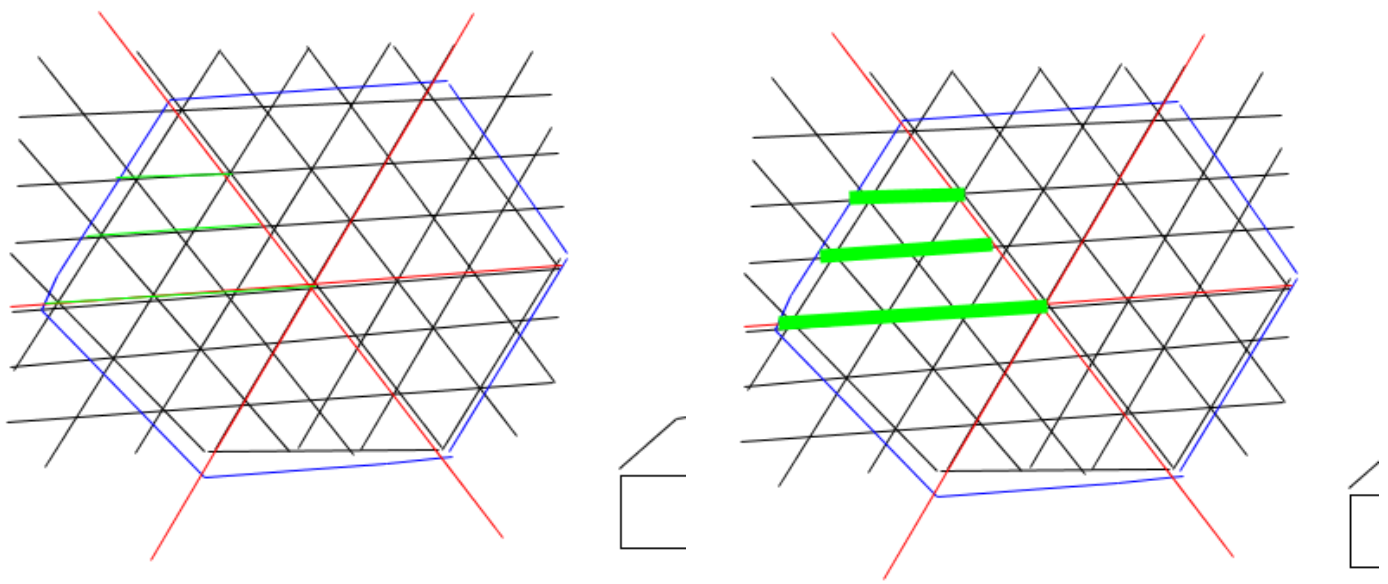

Figure 4: Qwertyuiop repositions the green lines on the left. Shortly after, 137 increases their thickness.

137's posting "the rows?" follows shortly after in line 750. The term "rows" has been previously used by this team to describe a method to systematically count the triangles located in one of the 6 regions of the hexagonal array earlier. By invoking this term here again, 137's posting proposes a relationship between what is highlighted on the drawing and a term the team has previously used to articulate a method of counting. The question mark appended invites others to make an assessment of the inferred relationship.

A minute after 137's question, Qwertyuiop posts a further elaboration. The first sentence states that the lines marked with green on the drawing are collinear to each other. The way he uses the term "collinear" here in relation to recently highlighted sticks indicates that this term is a reference to sticks that are aligned with respect to each other along a grid line. The second sentence asserts that there are " 3 identical sets of collinear lines" (presumably located within the larger triangular partition, since the green lines are carefully placed in such a partition). Finally, the last sentence states that one needs to find the number of sides (i.e., sticks) in one set and multiply that number by "3" (to find the total number of sticks in one partition). Although Qwertyuiop does not explicitly state it here, the way he places the green lines indicates that he is oriented to one of the 6 larger partitions to perform the counting operation he has just described. Following Qwertyuiop's elaboration, 137 posts “Ah. I see." in line 752. This is a token of cognitive change (Heritage, 2002), where the person who made the utterance announces that she/he can see something he has not been able to see earlier. Yet, it is still ambiguous what is understood or seen since no display of understanding is produced by the recipients yet.

Excerpt 2: Co-construction of a method for counting sticks

\begin{tabular}{|c|c|c|c|c|c|}
\hline $\begin{array}{l}\text { Chat } \\
\text { Index }\end{array}$ & $\begin{array}{l}\text { Time } \\
\text { Start } \\
\text { Typing }\end{array}$ & $\begin{array}{l}\text { Time of } \\
\text { Posting }\end{array}$ & Author & Content & Refers to \\
\hline \multirow[t]{2}{*}{752} & 19:30:20 & $19: 30: 23$ & 137 & Ah. I see. & \\
\hline & & $\begin{array}{l}19: 30: 48- \\
19: 30: 58\end{array}$ & & 137 drew an elongated hexagon in orange & \\
\hline 753 & 19:31:00 & 19:31:07 & 137 & Wait. Wouldn't that not work for that one? & \\
\hline 754 & 19:31:11 & 19:31:12 & Jason & Yeah & \\
\hline 755 & 19:31:12 & 19:31:15 & Jason & beacuse that's irregular & \\
\hline 756 & 19:31:09 & 19:31:17 & 137 & Or are we still only talking regular ones? & \\
\hline 757 & 19:31:20 & $19: 31: 22$ & 137 & About & \\
\hline 758 & 19:30:38 & $19: 31: 24$ & Qwertyuiop & $\begin{array}{l}\text { side length } 1=1 \text {, side length } 2=3 \text {, side length } 3 \\
=6 \ldots\end{array}$ & \\
\hline
\end{tabular}




\begin{tabular}{|c|c|c|c|c|c|}
\hline & & $\begin{array}{l}19: 31: 45- \\
19: 32: 15\end{array}$ & & 137 removes the orange hexagon & \\
\hline 759 & $19: 32: 32$ & $19: 32: 50$ & 137 & Shouldn't side length 2 be fore? & Message No. 758 \\
\hline 760 & $19: 32: 52$ & $19: 32: 53$ & 137 & ${ }^{*}$ four & \\
\hline 761 & $19: 33: 06$ & $19: 33: 10$ & Qwertyuiop & I count 3. & Message No. 759 \\
\hline 762 & $19: 33: 20$ & $19: 33: 25$ & 137 & Oh. Sry. & \\
\hline 763 & $19: 33: 24$ & 19:33:30 & Qwertyuiop & It's this triangle. & $\begin{array}{l}\text { Reference to } \\
\text { whiteboard (see } \\
\text { Figure 6) }\end{array}$ \\
\hline 764 & 19:33:44 & 19:33:45 & 137 & We & \\
\hline 765 & 19:33:47 & $19: 33: 54$ & Qwertyuiop & I don't see the pattern yet... & Message No. 758 \\
\hline \multirow[t]{2}{*}{766} & 19:33:50 & 19:34:01 & 137 & We're ignoring the bottom one? & \\
\hline & & $\begin{array}{l}19: 34: 10 \\
- \\
19: 34: 18\end{array}$ & & $\begin{array}{l}137 \text { first moves the longest green line, adds an } \\
\text { orange line segment, moves the longest line } \\
\text { back to its original position (see Figure 7) }\end{array}$ & \\
\hline 767 & $19: 34: 11$ & $19: 34: 29$ & Qwertyuiop & no, 3 is only for side length 2 . & Message No. 766 \\
\hline
\end{tabular}

About 18 seconds after 137's last posting, Qwertyuiop begins typing, but he does not post anything in chat for a while. After 10 seconds elapsed since Qwertyuiop started typing, 137 begins to produce a drawing on the whiteboard. In about 10 seconds, 137 produces a smaller hexagonal shape with orange color on the triangular grid. The new elongated hexagonal shape is placed on the right side of the recently added green lines, possibly to avoid overlap (see Figure 5). Once the hexagon is completed, 137 posts a chat message in line 753. The message starts with "wait" which can be read as an attempt to suspend the ongoing activity. The remaining part of the message states that the aforementioned approach may not work for a case indexed by the deictic term "that one". Since 137 has just recently produced an addition to the shared drawing, his message can be read in reference to the orange hexagon. Moreover, since the referred case is part of a message designed to suspend ongoing activity for bringing a potential problem to others' attention, the recently produced drawing seems to be presented as a counterexample to the current approach for counting the sticks.

\footnotetext{
${ }^{6}$ The token "wait" is used frequently in math problem-solving chats to suspend ongoing activity of the group and solicit attention to something problematic for the participant who uttered it. This token may be used as a preface to request explanation (e.g., wait a minute, I am not following, catch me up) or to critique a result or an approach as exemplified in this excerpt.
} 


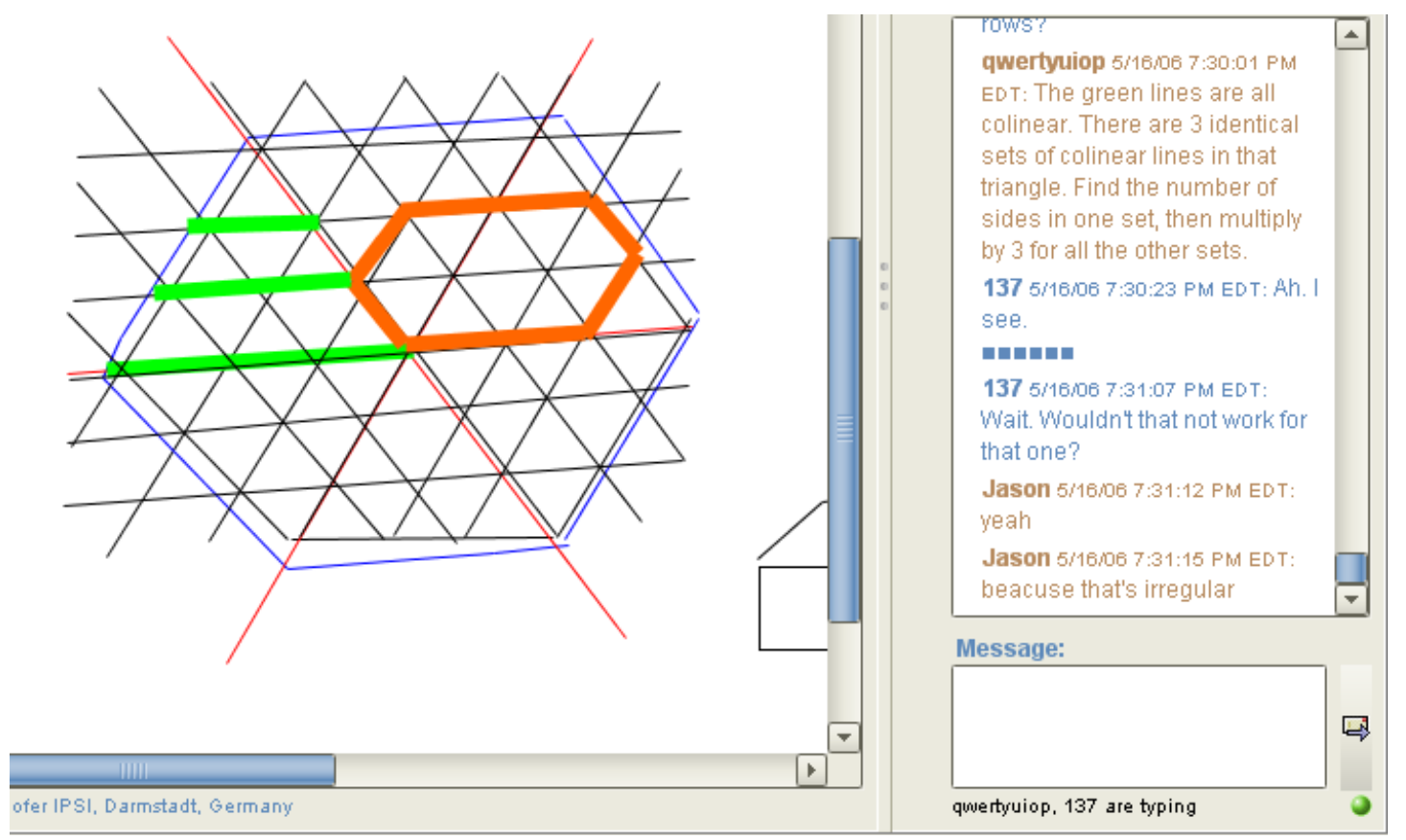

Figure 5: 137 adds an elongated hexagon in orange.

In the next line Jason posts the affirmative token "yes". Since it follows 137's remark sequentially, the affirmation can be read as a response to 137. Jason's following posting provides an account for the agreement by associating "irregularity" with an object indexed by the deictic term "that". When these two postings are read together in response to 137's message, the deictic term can be interpreted as a reference to the orange hexagon. In short, Jason seems to be stating that the strategy under consideration would not work for the orange hexagon because it is "irregular". In the meantime, 137 is still typing the statement that will appear in line 756, which asks whether the hexagon under consideration is still assumed to be regular. This question mitigates the prior problematization offered by the same author since it leaves the possibility that the proposed strategy by Qwertyuiop may still work for the regular case.

In line 758, Qwertyuiop posts a chat message stating "side length $1=1$, side length $2=3$, side length $3=$ 6..." It took about a minute for him to compose this message after he was first seen as typing at 19:30:38. The way the commas are used to separate the contents of the statement and the ellipsis placed at the end indicate that this posting should be read as an open-ended, ordered list. Within each list item the term "side length" is repeated. "Side length" has been used by this team during a prior session as a way to refer to different stages of a growing stick-pattern. In the hexagonal case the pattern has 6 sides at its boundary and counting by side-length means figuring out how many sticks would be needed to construct a given side as the pattern grows step by step. Note that this method of indexing stages assumes a stick-pattern that grows symmetrically. So a side length equal to 1,2 or 3 corresponds to the first, second or third stage of the hexagonal stick pattern, respectively. When the statement is read in isolation, it is not clear what the numbers on the right of the equals sign may mean, yet when this posting is read together with Qwertyuiop's previous posting where he described what needs to be found, these numbers seem to index the number of sticks within a set of collinear lines as the hexagonal array grows.

After Qwertyuiop's message, 137 removes the orange lines he has drawn earlier to produce an irregular hexagon. By erasing the irregular hexagon example, 137 seems to be taking Qwertyuiop's recent posting as a response to his earlier question posted in line 756, where he asked whether they were still considering regular hexagons or not. Although Qwertyuiop did not explicitly respond to this question, his message in line 758 (especially his use of the term "side length" which implicitly assumes such a regularity) seems to be seen as a continuation of the line of reasoning presented in his earlier postings. In other words, 
Qwertyuiop's sustained orientation to the symmetric case is taken as a response to the critique raised by 137.

In line 759, 137 posts a message explicitly linked to Qwertyuiop's most recent posting. It begins with the negative token "Shouldn't", which expresses disagreement. The subsequent "side length 2" indexes the problematic item and "be fore" offers a repair for that item. Moreover, the posting is phrased as a question to solicit a response from the intended recipient. 137's next posting in line 760 repairs his own statement with a repair notation peculiar to online chat environments. The asterisk at the beginning instructs readers to attend to the posting as a correction (usually to the most recent posting of the same author). In this case, due to its syntactic similarity to the word in the repair statement, "fore" seems to be the token that is supposed to be read as "four."

In his reply in line 761, Qwertyuiop insists that his counting yields "three" for the problematized case. In the next posting 137's "oh" marks the previous response as surprising or unexpected. The subsequent "sry - short for "sorry" - can be read as backing down. In line 763, Qwertyuiop posts a message that states "it's this triangle" and explicitly points at a region on the shared drawing. The explicit reference and the deictic terms again require the interlocutors to attend to something beyond the text involved in the posting. In short, the sequential unfolding of the recent postings suggests that this posting is designed to bring the relevant triangle in which the counting operation is done for the problematic case (indexed by side length 2) to other members' attention (see Figure 6).

In line 765, Qwertyuiop posts another message explicitly pointing to his earlier proposal for the first few values he obtained through his method of counting, where he states that he has not been able to "see a pattern yet." Hence, this statement explicitly specifies "the pattern" as what is missing or needed in this circumstance. The message not only brings a prospective indexical (Goodwin, 1996), "the pattern," into the ongoing discussion as a problem-solving objective, but also invites other members of the team to join the search for that pattern.

In the next line, 137 posts a question that brings other members' attention to something potentially ignored so far. The term "bottom one" when used with "ignore" indexes something excluded or left out. Nine seconds after his posting, 137 performs some drawing work on the whiteboard. He moves the longest green line to the right first, then he adds a short line segment with orange color, and then he moves the same green line back to its original location (see Figure 7). These moves make 137's orientation to a particular part of the drawing explicit. When read together with his previous question, the orange line could be seen as a graphical illustration for the left-out part previously referred as the "bottom one". When read as a response to Qwertyuiop's recent exposition in lines 761 and 763, the "bottom one" seems to be a reference to the part of the drawing that was not enclosed by Qwertyuiop's explicit reference.

\footnotetext{
${ }^{7}$ Goodwin (1996) proposes the term prospective indexicals for those terms whose sense is not yet available to the participants when it is uttered, but will be discovered subsequently as the interaction unfolds. Recipients need to attend to the subsequent events to see what constitutes a "pattern" in this circumstance.
} 


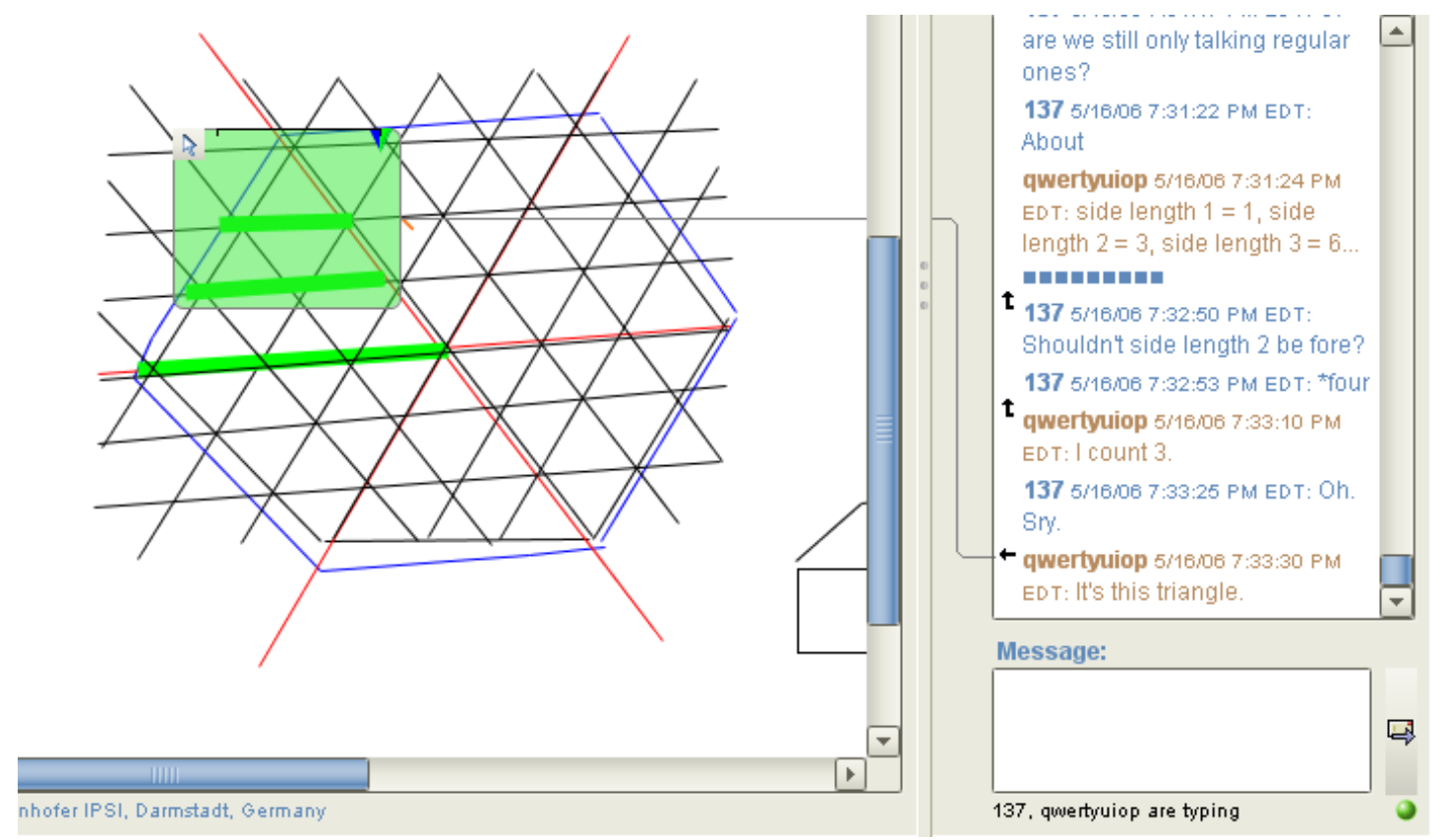

Figure 6: Qwertyuiop points to the triangle which contains the sticks to be counted for the stage indexed by side length $=2$. The green lines enclosed by the reference correspond to $1+2=3$ sticks.

The next posting by Qwertyuiop, which appears in line 767, is explicitly linked to 137's question in the previous line. The message begins with "no" which marks the author's disagreement with the linked content, and the subsequent part of the message provides an account for the disagreement by stating that the value 3 is only relevant to the case indexed by "side length 2".

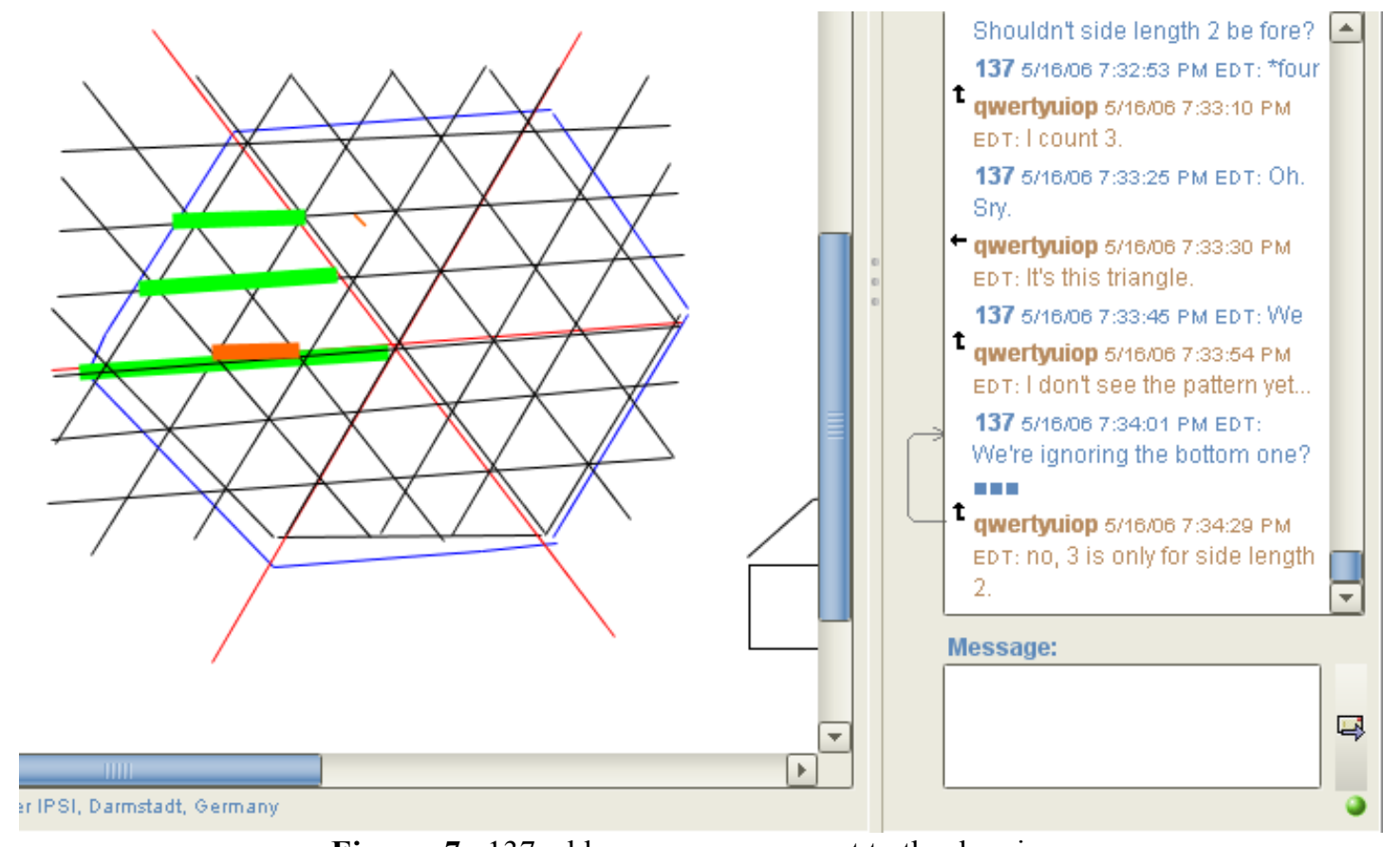

Figure 7: 137 adds an orange segment to the drawing.

The sequence of exchanges between 137 and Qwertyuiop in this excerpt indicates that there is a misalignment within the group about the procedure used for counting the number of sticks. This 
misalignment is made evident through explicit problematizations and disagreements. The way the members make use of both spaces as they interact with each other makes it increasingly clear for them (a) where the relevant pieces indexed by the terms like "collinear" and "triangle" are located, and (b) how they are used in the counting process. Nevertheless, the misalignment between the counting procedures suggested in 137's and Qwertyuiop's contributions have not been resolved yet.

\section{Excerpt 3: Collective noticing of a pattern of growth}

\begin{tabular}{|c|c|c|c|c|c|}
\hline $\begin{array}{l}\text { Chat } \\
\text { Index }\end{array}$ & $\begin{array}{l}\text { Time } \\
\text { Start } \\
\text { Typing }\end{array}$ & $\begin{array}{l}\text { Time of } \\
\text { Posting }\end{array}$ & Author & Content & Refers to \\
\hline 765 & 19:33:47 & 19:33:54 & Qwertyuiop & I don't see the pattern yet... & Message No. 758 \\
\hline \multirow[t]{2}{*}{766} & $19: 33: 50$ & 19:34:01 & 137 & We're ignoring the bottom one? & \\
\hline & & $\begin{array}{l}19: 34: 10- \\
19: 34: 18\end{array}$ & & $\begin{array}{l}137 \text { first moves the longest green line, adds } \\
\text { an orange line segment, moves the longest } \\
\text { line back to its original position }\end{array}$ & \\
\hline 767 & 19:34:11 & $19: 34: 29$ & Qwertyuiop & No, 3 is only for side length 2 . & Message No. 766 \\
\hline \multirow[t]{2}{*}{768} & 19:34:36 & $19: 34: 52$ & 137 & $\begin{array}{l}\text { And I think the'y;re all triangular } \\
\text { numbers. }\end{array}$ & Message No. 765 \\
\hline & & $\begin{array}{l}19: 35: 03- \\
19: 35: 16\end{array}$ & & $\begin{array}{l}137 \text { 's changes the color of the longest green } \\
\text { line to red, and then to green again }\end{array}$ & \\
\hline \multirow[t]{2}{*}{769} & 19:35:06 & 19:35:17 & Qwertyuiop & "triangular numbers"? & Message No. 768 \\
\hline & & $\begin{array}{l}19: 35: 27- \\
19: 35: 36\end{array}$ & & $\begin{array}{l}\text { 137's draws a red hexagon on the diagram } \\
\text { (Figure 8) }\end{array}$ & \\
\hline 770 & $19: 35: 28$ & $19: 35: 37$ & Jason & You mean like $1,3,7, \ldots$ & \\
\hline 771 & 19:35:39 & 19:35:39 & Jason & $?$ & \\
\hline 772 & $19: 35: 48$ & 19:35:59 & 137 & Like 1,3,6,10,15,21,28. & Message No. 770 \\
\hline 773 & 19:35:51 & 19:36:02 & Qwertyuiop & The sequence is $1,3,6 \ldots$ & Message No. 770 \\
\hline 774 & 19:36:02 & 19:36:30 & 137 & $\begin{array}{l}\text { Numbers that can be expressed as } \\
\mathrm{n}(\mathrm{n}+1) / 2 \text {, where } \mathrm{n} \text { is an integer. }\end{array}$ & \\
\hline 775 & $19: 36: 44$ & $19: 36: 45$ & Qwertyuiop & $\mathrm{Ah}$ & \\
\hline 776 & 19:37:09 & 19:37:18 & 137 & $\begin{array}{l}\text { So are we ignoring the bottom orange line } \\
\text { for now? }\end{array}$ & Message No. 766 \\
\hline
\end{tabular}

In line 768, 137 posts a message linked to Qwertyuiop's posting in line 765. The preface "And" and the explicit reference together differentiate this contribution from the ongoing discussion about a piece that was potentially excluded from the second stage. Note that Qwertyuiop's message in line 765 refers further back to an older posting where he proposed a sequence of numbers for the first 3 stages "side length $1=$ 1 , side length $2=3$, side length $3=6 \ldots$.." When 137's message is read in relation to these two prior messages, the phrase "they are all" seems to be a reference to this sequence of numbers. Therefore, the message can be read as an uptake of the issue of finding a pattern that fits this sequence. Moreover, by proposing the term "triangular numbers" as a possible characterization for the sequence, 137 offers further specificity to the prospective indexical, the "pattern", which was initially brought up by Qwertyuiop.

Following his proposal, 137 changes the color of the longest green line segment at the bottom to red and then to green again. In the meantime Qwertyuiop is typing what will appear in line 769, which can be read as a question soliciting further elaboration of the newly contributed term "triangular numbers." 137 
continues to act on the whiteboard and he adds a red hexagon to the shared drawing (see Figure 8). Since the hexagon is located on the section referenced by Qwertyuiop several times earlier and shares an edge with the recently problematized orange section, this drawing action can be treated as a move related to the discussion of the ignored piece.

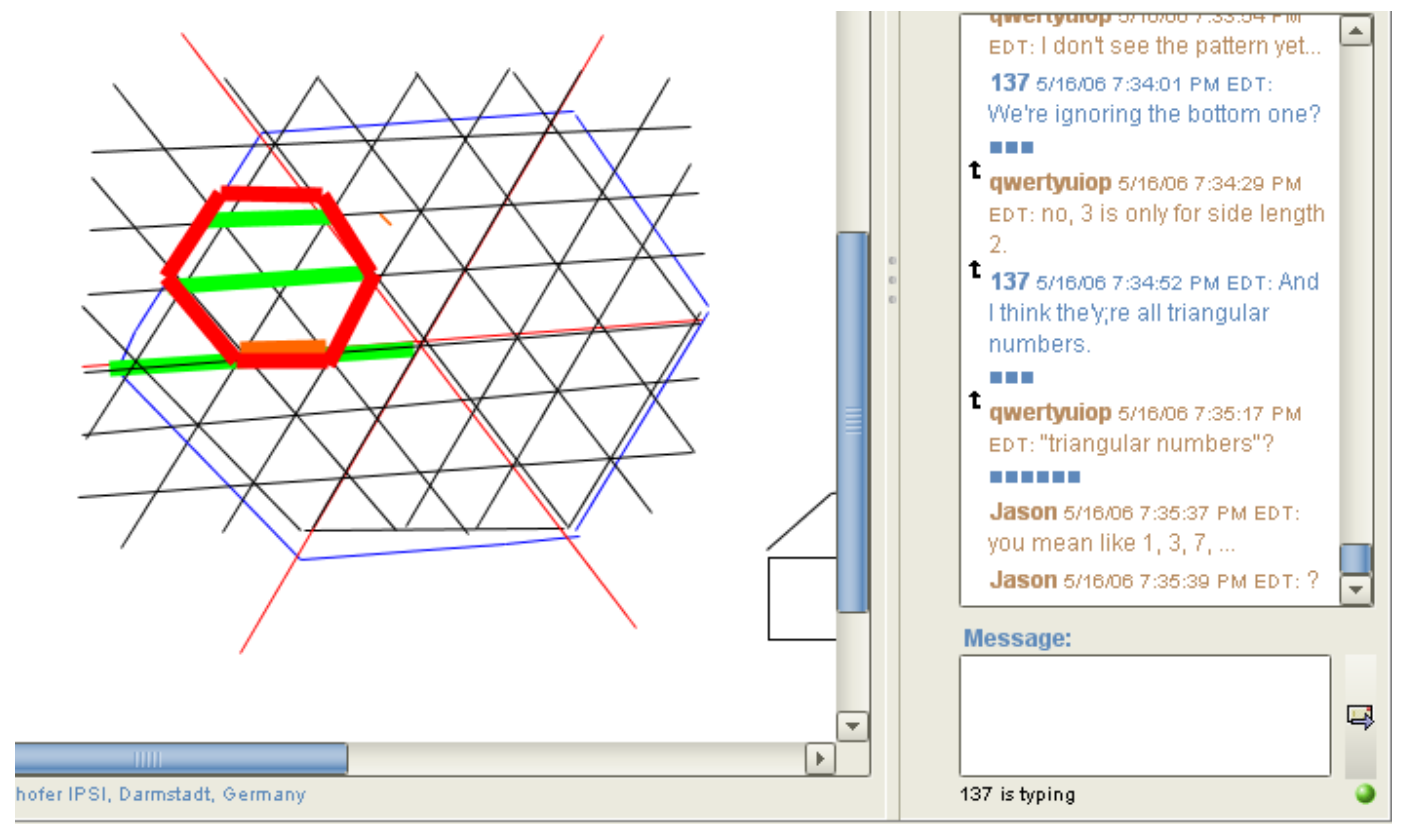

Figure 8: 137 adds a red hexagon inside the partition the team has been oriented to.

Jason joins the discussion thread about triangular numbers by offering a list of numbers in line 770 . The term "like" is used here again to relate a mathematical term to what it may be indexing. This posting alone can be read as an assertion, but the question mark Jason posts immediately after in the next line mitigates it to a statement soliciting others' assessment. At roughly the same time, 137 posts a substantially longer sequence of numbers, and immediately after Qwertyuiop points out the difference between 137's sequence and what Jason offered as a list of triangular numbers. In line 774, 137 elaborates his definition further by offering an algebraic characterization of triangular numbers as integers that can be expressed with the formula " $n(n+1) / 2$ ".

In short, the sequence resulting from Qwertyuiop's counting work based on his notion of "collinearity" has led the team to notice a relationship between that sequence and a mathematical object called "triangular numbers". The latter symbolic definition offered by 137 for triangular numbers in response to the ongoing search for a pattern has established a relationship between geometrically motivated counting work and an algebraic/symbolic representation stated in generic form as $\mathbf{n}(\mathbf{n + 1}) / 2$.

\section{Excerpt 4: Resolution of referential ambiguity via visual proof}

\begin{tabular}{|l|l|l|l|l|l|}
\hline $\begin{array}{l}\text { Chat } \\
\text { Index }\end{array}$ & $\begin{array}{l}\text { Time } \\
\text { Start } \\
\text { Typing }\end{array}$ & $\begin{array}{l}\text { Time of } \\
\text { Posting }\end{array}$ & Author & Content & Refers to \\
\hline 776 & $19: 37: 09$ & $19: 37: 18$ & 137 & $\begin{array}{l}\text { So are we ignoring the bottom orange } \\
\text { line for now? }\end{array}$ & Message No. 766 \\
\hline 777 & $19: 37: 32$ & $19: 37: 36$ & Qwertyuiop & "green"? & Message No. 776 \\
\hline 778 & $19: 37: 44$ & $19: 37: 48$ & 137 & THe short orange segment. & \\
\hline & & $19: 37: 59-$ & & $\mathbf{1 3 7}$ changes the color of the green lines & \\
\hline
\end{tabular}




\begin{tabular}{|c|c|c|c|c|c|}
\hline & & 19:38:02 & & $\begin{array}{l}\text { enclosed by the red hexagon to blue (see } \\
\text { Figure 9) }\end{array}$ & \\
\hline 779 & 19:37:49 & 19:38:05 & 137 & PArallel to the blue lines. & \\
\hline 780 & 19:37:58 & 19:38:05 & Qwertyuiop & I don't think so... & \\
\hline 781 & 19:38:20 & 19:38:26 & 137 & $\begin{array}{l}\text { Wait, we are counting sticks right now, } \\
\text { right? }\end{array}$ & Message No. 780 \\
\hline 782 & 19:38:35 & 19:38:48 & Qwertyuiop & yes-one of the colinear ets of sticks & \\
\hline 783 & 19:38:55 & 19:39:08 & Qwertyuiop & oops-"sets" not " ets" & \\
\hline 784 & 19:39:22 & 19:39:42 & 137 & $\begin{array}{l}\text { So we are trying to find the total number } \\
\text { of sticks in a given regular hexagon? }\end{array}$ & Message No. 782 \\
\hline 785 & 19:39:50 & 19:40:18 & Qwertyuiop & $\begin{array}{l}\text { not yet-we are finding one of the three } \\
\text { sets, then multiplying by } 3\end{array}$ & Message No. 784 \\
\hline 786 & 19:40:25 & 19:40:40 & Qwertyuiop & $\begin{array}{l}\text { that will give the number in the whol } \\
\text { triangle }\end{array}$ & \\
\hline 787 & 19:40:34 & 19:40:51 & 137 & $\begin{array}{l}\text { Then shouldn't we also count the bottom } \\
\text { line? }\end{array}$ & Message No. 785 \\
\hline 788 & 19:40:52 & 19:41:01 & Jason & $\begin{array}{l}\text { are you taking into account the fact that } \\
\text { some of the sticks will overlap }\end{array}$ & Message No. 786 \\
\hline 789 & 19:41:25 & 19:41:41 & 137 & $\begin{array}{l}\text { Then number of sticks needed for the } \\
\text { hexagon, right? }\end{array}$ & Message No. 786 \\
\hline 790 & 19:41:16 & 19:42:22 & Qwertyuiop & $\begin{array}{l}\text { Yes. The blue and green/orange lines } \\
\text { make up on of the three colinear sets of } \\
\text { sides in the triangle. Each set is identical } \\
\text { and doesn't overlap with the other sets. }\end{array}$ & Message No. 788 \\
\hline 791 & 19:42:50 & 19:42:50 & Jason & Ok & \\
\hline 792 & 19:43:03 & 19:43:11 & Jason & $\begin{array}{l}\text { this would be true for hexagons of any } \\
\text { size right> }\end{array}$ & \\
\hline 793 & 19:43:09 & $19: 43: 13$ & Qwertyuiop & triangle, so far & Message No. 789 \\
\hline 794 & 19:43:25 & 19:43:25 & 137 & Oh. & \\
\hline 795 & 19:43:25 & 19:43:26 & Qwertyuiop & this one & $\begin{array}{l}\text { Reference to } \\
\text { whiteboard (see } \\
\text { Figure 10) }\end{array}$ \\
\hline 796 & 19:43:42 & $19: 43: 52$ & 137 & Yes, but they will overlap... & \\
\hline 797 & 19:43:59 & $19: 44: 13$ & 137 & $\begin{array}{l}\text { Eventually when you multiply by } 6 \text { to get } \\
\text { it for the whole figure. }\end{array}$ & \\
\hline \multirow[t]{2}{*}{798} & 19:44:01 & 19:44:30 & Qwertyuiop & $\begin{array}{l}\text { no, the sets are not collinear with } \\
\text { eachother. I'll draw it... }\end{array}$ & Message No. 796 \\
\hline & & $\begin{array}{l}\text { 19:44:35- } \\
19: 44: 56\end{array}$ & & $\begin{array}{l}\text { Qwertyuiop moves the small hexagon in } \\
\text { red and blue lines out of the grid (see } \\
\text { Figure 11) }\end{array}$ & \\
\hline \multirow[t]{5}{*}{799} & & 19:44:59 & 137 & & Message No. 798 \\
\hline & & $\begin{array}{l}\text { 19:44:59- } \\
\text { 19:45:17 }\end{array}$ & & $\begin{array}{l}\text { Qwertyuiop repositions and resizes the } \\
\text { red lines on the grid }\end{array}$ & \\
\hline & & 19:45:20 & & $\begin{array}{l}\text { Qwertyuiop continues adjusting the red } \\
\text { lines }\end{array}$ & \\
\hline & & $\begin{array}{l}19: 45: 23- \\
19: 45: 37\end{array}$ & & $\begin{array}{l}\text { Qwertyuiop continues adjusting the red } \\
\text { lines }\end{array}$ & \\
\hline & & $\begin{array}{l}\text { 19:45:41- } \\
19: 46: 16\end{array}$ & & $\begin{array}{l}\text { Qwertyuiop adds purple lines (see Figure } \\
\text { 12) }\end{array}$ & \\
\hline
\end{tabular}




\begin{tabular}{|c|c|c|c|c|c|}
\hline 800 & 19:46:22 & 19:46:34 & 137 & Oh. I see. & \\
\hline \multirow[t]{2}{*}{801} & $19: 46: 22$ & $19: 46: 52$ & Qwertyuiop & $\begin{array}{l}\text { Those are the } 3 \text { sets. One is red, one is } \\
\text { green, one is purple. }\end{array}$ & \\
\hline & & $\begin{array}{l}19: 47: 07- \\
19: 47: 11\end{array}$ & 137 & 137 starts to make green lines thicker & \\
\hline \multirow[t]{2}{*}{802} & 19:47:04 & $19: 47: 12$ & Jason & wait--- i don't see the green/purple ones & \\
\hline & & $\begin{array}{l}19: 47: 17- \\
19: 47: 33\end{array}$ & 137 & $\begin{array}{l}137 \text { makes the purple lines thicker (see } \\
\text { Figure } 13 \text { below) }\end{array}$ & \\
\hline 803 & $19: 47: 18$ & $19: 47: 40$ & Qwertyuiop & $\begin{array}{l}\text { so we find a function for that sequence } \\
\text { and multiply by } 3\end{array}$ & Message No. 774 \\
\hline
\end{tabular}

In line 776, 137 posts a message which is explicitly linked to his prior message in line 766 where he mentioned a potentially ignored piece indexed by the phrase "the bottom one". The use of "So" at the beginning can be read as an attempt to differentiate this message from the recently unfolding discussion about triangular numbers. The subsequent part of the message brings other team members' attention to a potentially ignored piece indexed by the phrase "the bottom orange line". 137 used the phrase "the bottom one" earlier, but this time he makes use of color referencing as an additional resource to provide further specificity to what he is referencing. At this moment a red hexagon and a short orange segment are visible on the shared drawing space, which are layered on top of the triangular grid (see Figure 8). The way 137 orients to the new state of the drawing indicates that his earlier drawing actions (marked in the prior excerpt before line 770) seem to be performed in preparation for this posting. Hence, this posting can be read as an attempt to re-initiate a prior thread about a potentially ignored piece in the counting work, which is distributed over both interaction spaces.

Qwertyuiop's message in the next line involves "green" in quotes, ends with a question mark, and is explicitly linked to 137 's last message in line 776. The quotation marks seem to give significance to an object indexed by the color reference. Note that there are 3 green lines on the shared drawing at the moment (see Figure 8). The use of the color reference and the explicit link suggest that this message is posted in response to 137's question in line 776. When it is read in this way, Qwertyuiop seems to be asking if the relevant line located at the bottom should have been the green one instead.

Following Qwertyuiop's posting, 137 provides further specificity to the problematized object by first stating that it is "the short orange segment" in line 778. Next, 137 modifies the two green lines inside the red hexagon by changing their color to blue (see Figure 9). Then, he posts another message in line 779 that refers to a particular location on the whiteboard that is "parallel" to the recently added "blue lines". Thus, 137's recent actions suggest that the object indexed by his phrase, "short bottom orange line" segment, is the one parallel to the blue lines. 


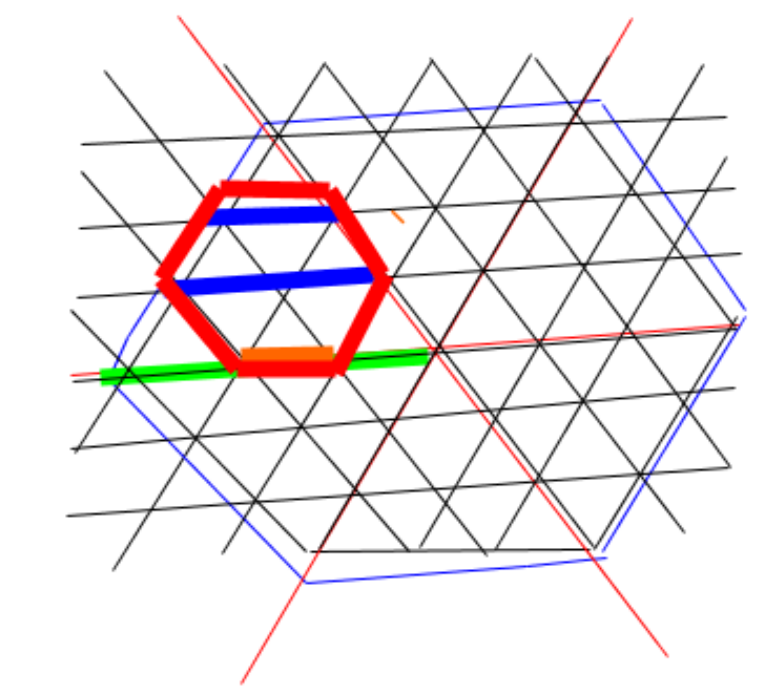

ofer IPSI, Darmstadt, Germany

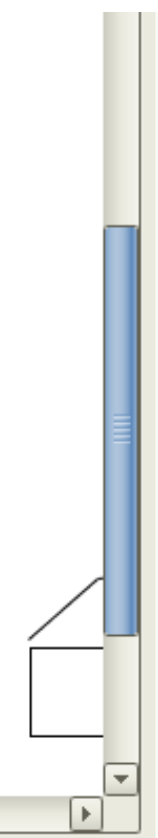

Figure 9: 137 changes the color of the green lines inside the red hexagon to blue

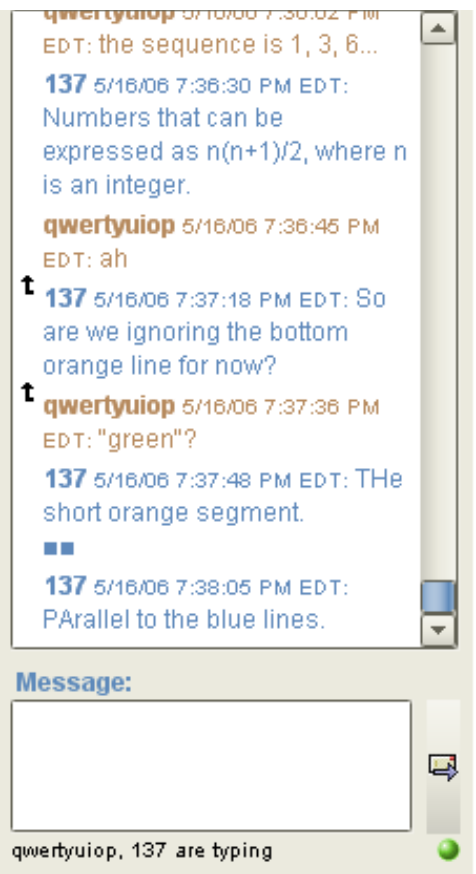

EDT: the sequence is $1,3,6$.

(

is an integer.

qwertyuiop 5/16:06 7:36:45 PM

are we ignoring the bottom

EDT: "green"?

137 5/16/06 7:37:48 PM EDT: THe short orange segment.

(1⿴囗十)

137 5/16/06 7:38:05 PM EDT: PArallel to the blue lines.

In line 780, Qwertyuiop states his disagreement. Since the message appears shortly after 137's point that the orange segment is left out of the computation, Qwertyuiop seems to be disagreeing with the remark that there is a missing piece in the counting method. In the next line, 137 posts a question prefaced with "wait" that calls for suspending the ongoing activity and asks if one can still characterize what the team ("we") is currently doing as "counting the sticks". The posting is explicitly linked to Qwertyuiop's last message. By posting a question about the ongoing group process following a sustained disagreement with his peer, 137 is making it explicit that there is a misalignment within the team with respect to the task at hand. Hence, this exchange marks a breakdown in interaction that needs to be attended to before the team can proceed any further.

In the next line, Qwertyuiop takes up this question by providing his account of the ongoing process as counting "one of the collinear sets of sticks." Next, 137 posts another question explicitly linked to Qwertyuiop's answer, which gives further specificity to 137's earlier characterization of the counting work undertaken by the team (i.e., counting the sticks for the "whole hexagon"). Qwertyuiop's response to this question states that the focus is not on the whole hexagon yet, but on what he is referring to as "one of the three sets", which would then be followed by a multiplication by 3 . In the next line Qwertyuiop continues his explanation that this will give them the number of sticks for "the whole triangle", which can be read as a reference to one of the six triangular partitions that altogether form the hexagon.

In line 787, 137 posts a message explicitly linked to the first part of Qwertyuiop's explanation. The posting is phrased as a question problematizing again that the bottom line should also be included in the counting operation described by Qwertyuiop. Next, Jason joins the discussion by posting a question linked to the latter half of Qwertyuiop's explanation in line 786, which asks him if he has taken into account "the fact that some of the sticks will overlap". The way Jason phrases his posting brings "overlap" as an issue that needs to be addressed by the counting method under discussion.

In line 789, 137 posts a chat message with a referential link to Qwertyuiop's last posting in line 786. This message seems to extend the order of computations described in Qwertyuiop's exposition by anticipating the next step of the computation, namely calculating the number of sticks needed for the hexagon once the 
step mentioned in 786 is achieved. In other words, 137 displays that he is able to follow the order of computations suggested by his peer to address the task at hand.

In line 788 Qwertyuiop responds to the overlapping sticks issue raised by Jason. He makes reference to the blue and green/orange lines to describe one of the three collinear sets of sides within the triangular partition (since the shared image has remained unchanged, this message can be read in reference to the state displayed in Figure 9). He further asserts that each set is identical and does not overlap. In the next line Jason concurs, and then asks if this should hold for hexagons of any size.

Following Jason's messages, Qwertyuiop posts a message linked to 137's earlier question in line 789. Qwertyuiop stresses again that the focus has been on the "triangle" so far. His next posting in line 795 includes a referential arrow to the shared diagram and a deictic term "this one" that together provide further specificity to which part of the hexagon he was referring to with the indexical term "triangle" (see Figure 10).

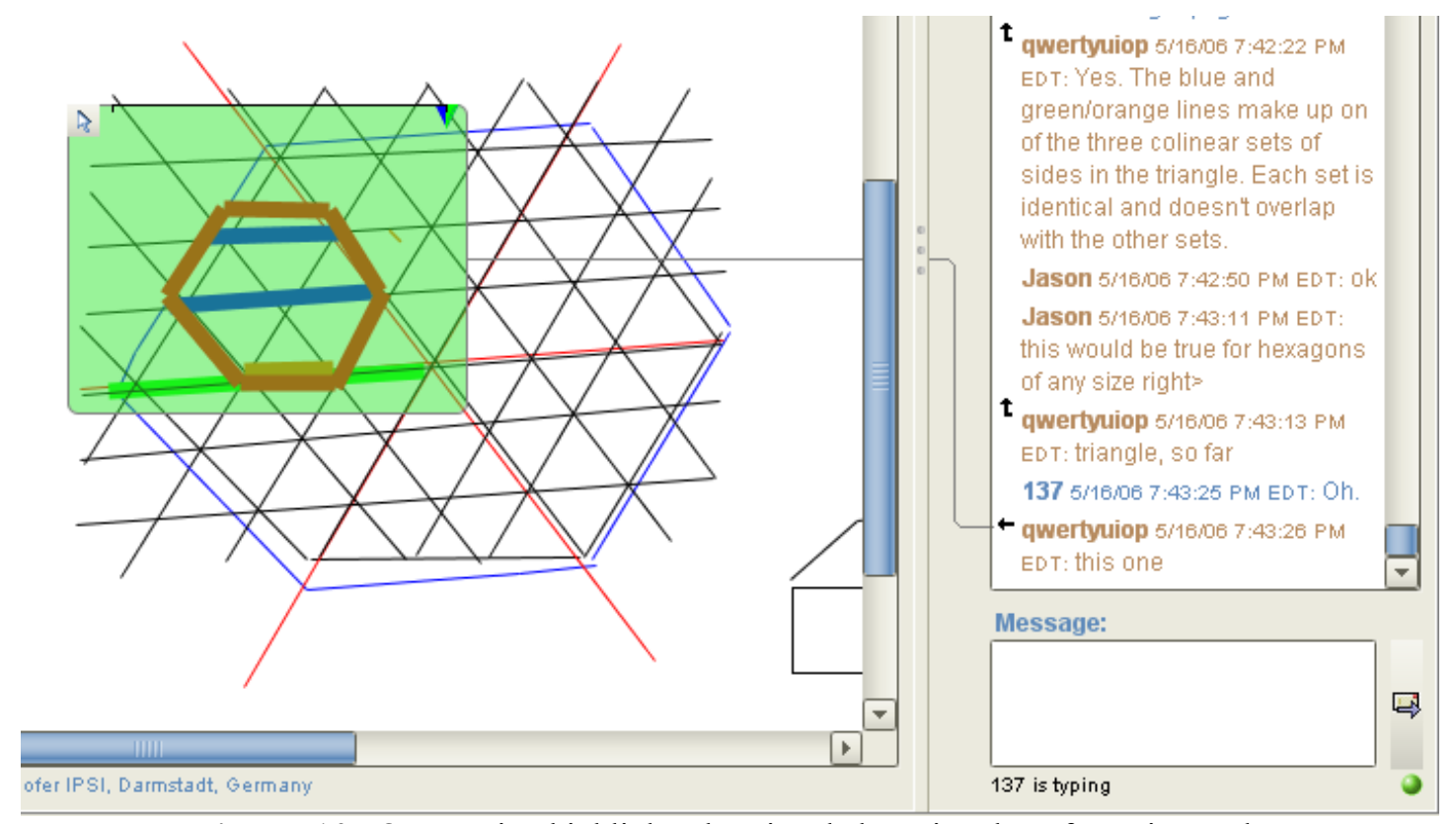

Figure 10: Qwertyuiop highlights the triangle by using the referencing tool.

In lines 796 and 797, 137 first accepts what Qwertyuiop has asserted, but points to a potential issue that will be faced when the result will be multiplied by 6 to extend the counting operation to the whole hexagon. Before 137 posts his elaboration in line 797, Qwertyuiop begins typing a response to 137's first remark that appears in line 798. In that message Qwertyuiop expresses his disagreement and asserts that "the sets are not collinear with each other". Hence, this posting shows that Qwertyuiop has treated 137's use of the pronoun "they" in line 796 as a reference to the notion of collinear sets. In the latter part of his posting, Qwertyuiop announces that he will draw what he is talking about, so this section of the message projects that a related drawing action will follow his statement shortly. 


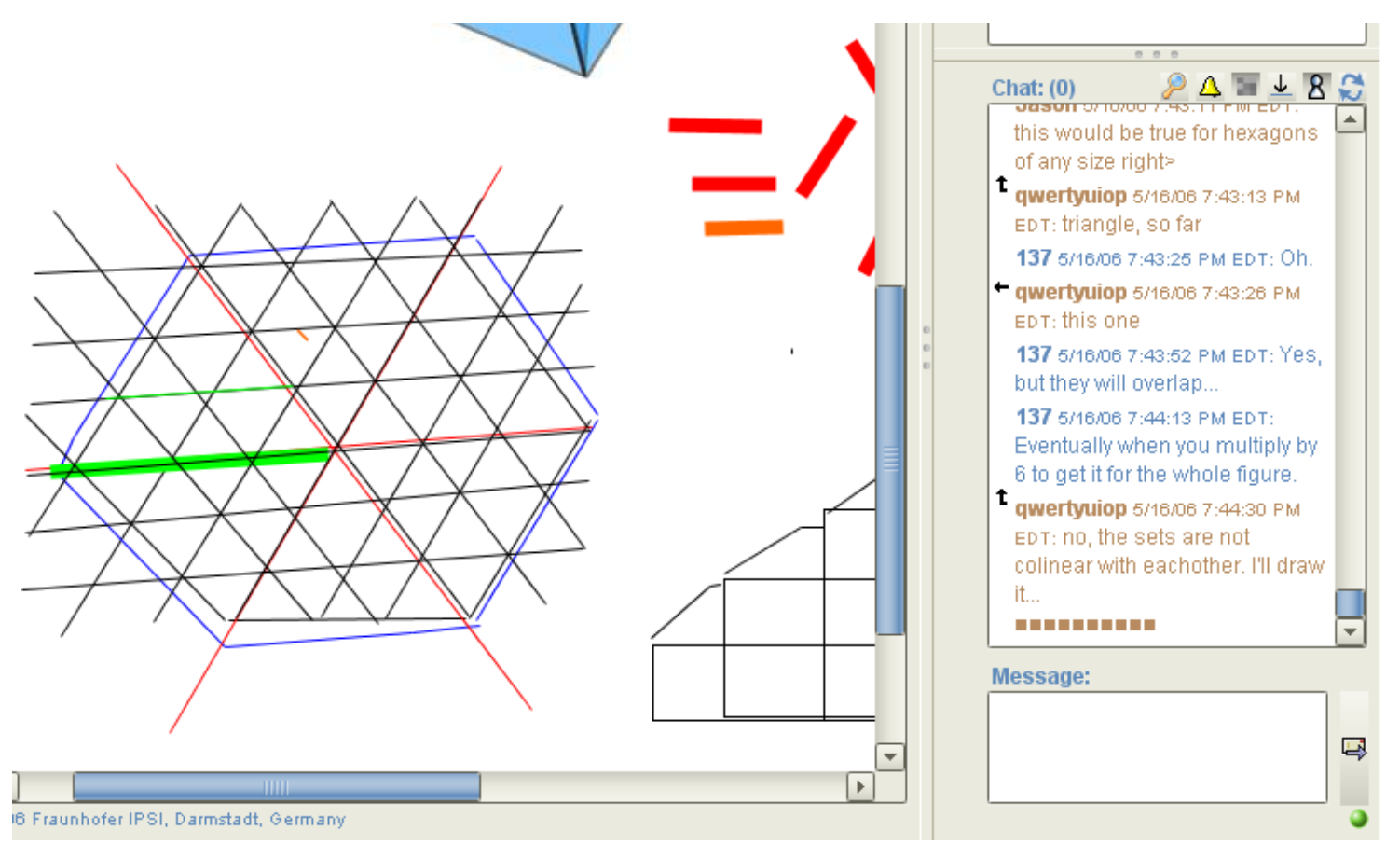

Figure 11: Qwertyuiop moves the lines added by 137 away.

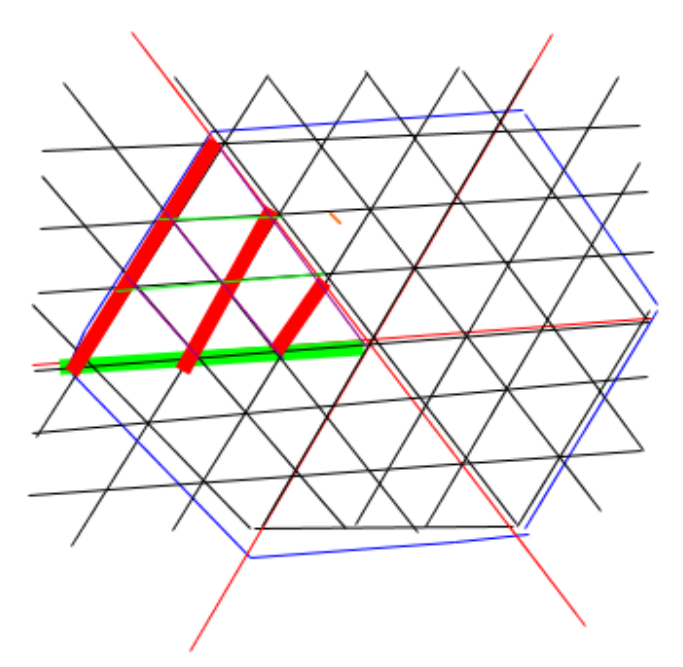

$\frac{1}{36 \text { Fraunhofer IPSI, Darmstadt, Germany }}$

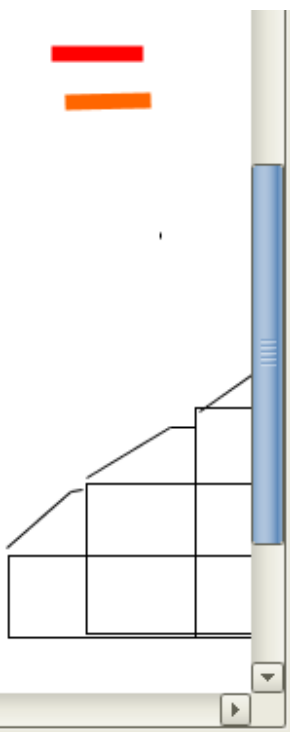

Figure 12: Qwertyuiop repositions the red lines to mark a part of the larger triangle. Then he adds two horizontal lines in green, parallel to the existing green line. Finally, he adds 3 more lines in purple. Since Qwertyuiop uses a thinner brush to draw the green and purple lines, they are difficult to see.

Figures 11 and 12 display snapshots from Qwertyuiop's drawing actions following his last posting. First he moves the red and orange lines to the side, and then he repositions the red lines to highlight 3 segments that are parallel to each other. Next, he adds 2 green lines parallel to the remaining green line. Finally, he adds 3 purple lines to cover the remaining sticks in that triangular section. The green and purple lines are drawn with a thin brush (see Figure 12). 
Once the drawing reaches the stage in Figure 12, 137 posts "oh I see" in line 800, which can be read in response to Qwertyuiop's recent drawing work. Qwertyuiop's graphical illustration seemed to have helped 137 to notice something he had not been able to see earlier. Next, Qwertyuiop posts a message that refers to the lines he has recently drawn with the plural deictic term "those". The message provides further specificity to the mathematics object " 3 sets" by locating each set on the diagram through the use of color references "red", "green" and "purple". In other words, Qwertyuiop has provided a visual realization of the phrase " 3 sets of collinear sides" he coined earlier, which has been treated as problematic by his teammates.

In line 802, Jason states that he cannot see the green/purple lines, which were marked with a thin brush by Qwertyuiop. In response 137 makes these new additions more visible by increasing their thickness (see Figure 13). The final state of the diagram presents a visual proof that 3 sets of collinear lines marked with green, purple, and red do not indeed overlap with each other.

In line 803, Qwertyuiop provides further specificity as to what needs to be found given the visual realization of the collinear sides recently produced on the whiteboard. His message is explicitly linked to an old message posted by 137 several lines ago (line 774 in Excerpt 3) that provides a formulaic realization for triangular numbers previously associated with the pattern of growth of collinear sides. Hence, Qwertyuiop's statement, "find a function for that sequence and multiply by 3", can be read as a proposal for a strategy to find the number of sticks required to build a triangular partition. In particular, Qwertyuiop is pointing (narratively) to a candidate (symbolic) algebraic realization of what he has just demonstrated with (graphical) visual resources on the whiteboard. This is the culmination of a sublte and complex collaborative process in which mathematical discourse, graphical reasoning and symbolic expression were tightly integrated by the group.

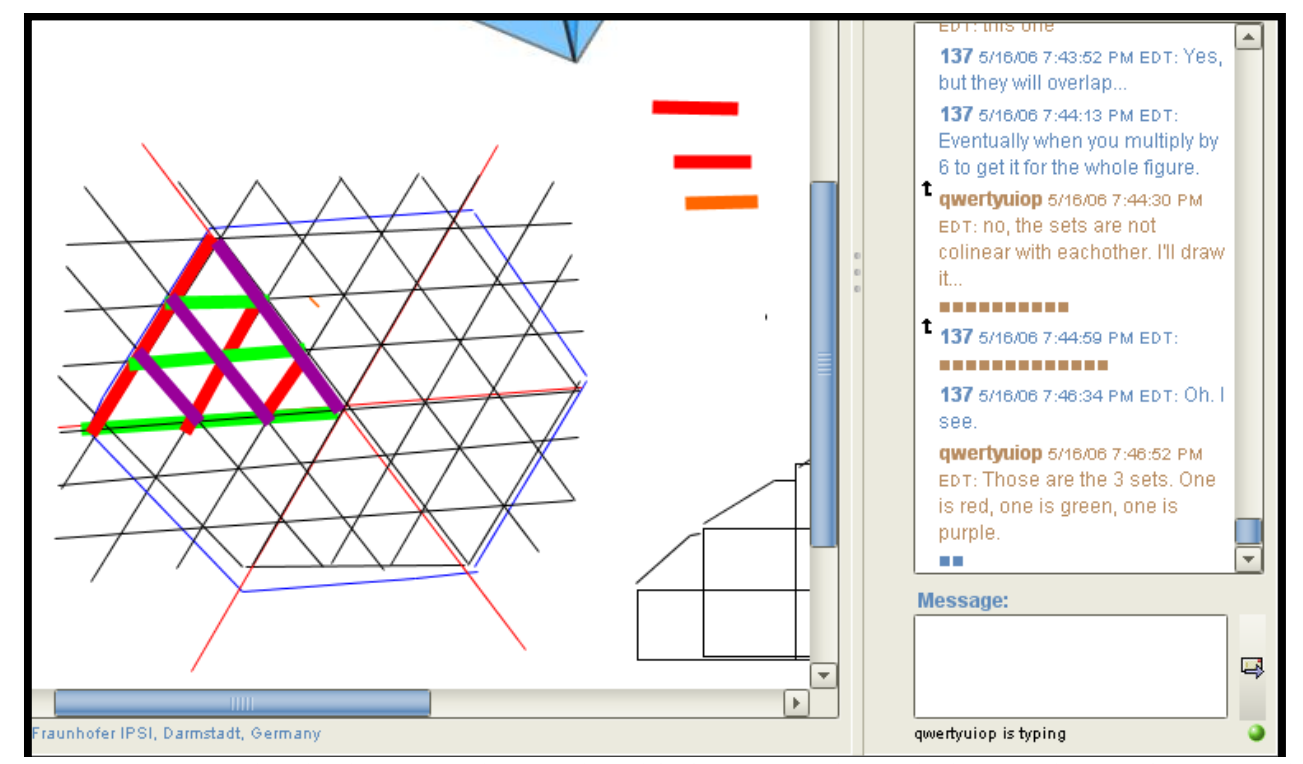

Figure 13: 137 increases the thickness of the newly added green and purple lines. The final state of the diagram presents a visual proof that 3 sets of collinear lines do not overlap with each other.

To sum up, in this episode the team has achieved a sense of common ground (Clark \& Brennan, 1991), intersubjectivity (Stahl, et al., 2011) or indexical symmetry ${ }^{8}$ (Hanks, 1992; 2000) with respect to the term

\footnotetext{
${ }^{8}$ Hanks proposes the notion of indexical symmetry to characterize the degree to which the interactants share, or fail to share, a common framework relative to some field of interaction on which reference can be made. In particular, "...the more interactants share, the more congruent, reciprocal and transposable their perspectives, the more symmetric is the interactive field. The greater the differences that divide them, the more asymmetric the field."
} 
"set of collinear sides" and its projected application towards solving the task at hand. The challenges voiced by 137 and Jason through the course of the episode solicited further elaboration from Qwertyuiop regarding how collinear sides can be located in the shared diagram and how they can be used to devise a method to count the number of sticks. In particular, in this excerpt the team members worked out the overall organization of their joint problem-solving work by discussing what they are trying to find, how they should locate the objects relevant to the task, and how they should order some of the steps that have been proposed so far to arrive at a solution. For instance, Qwertyuiop's initial proposal including the indexical term "collinear sets" focuses on one of the triangular regions. Yet, the focus on a triangular region was left implicit, which seemed to have led 137 to treat Qwertyuiop's proposal as applied to the whole hexagon. Through their discussion across both interaction spaces the team has incrementally achieved a shared understanding in terms of how a triangular region is decomposed into 3 sets of collinear, non-overlapping sides, and how that can be used to systematically count the number of sticks in that region. The visual practices have been encapsulated in linguistic terms in ways that become shared within the small group through their interactions, which integrate graphical and narrative actions. The graphical moves are strategically motivated to decompose a complicated pattern into visually obvious sub-patterns, with an eye to subsequently constructing a symbolic representation of the pattern. The elaboration of a mathematical vocabulary allows the group to reference the elements of their analysis in order to establish a shared view of the graphical constructions, to make proposals about the patterns to each other and to index past established results.

\section{Concluding the Mathematical Analysis}

The group is now ready to return to the symbolic work. In line $818^{9}$, Qwertyuiop resumes the discussion about the shared task by proposing a formula " $f(n)=2 n-1$ " where he declares $n$ to be the "side length" (see Excerpt 5). It is not evident from the text itself what the formula is standing for. Yet, the message is explicitly linked to an older posting (line 772) where 137 posted the statement "Like 1,3,6,10,15,21,28" as part of a prior discussion on triangular numbers (see Excerpt 3). Hence, when this message is read in reference to line 772, it can be treated as a proposal to generalize the values derived from Qwertyuiop's geometrically informed counting method with a formula stated in symbolic form.

\begin{tabular}{|c|c|c|c|c|c|}
\hline \multicolumn{6}{|c|}{ Excerpt 5: Re-initiating the discussion of the algebraic formula } \\
\hline $\begin{array}{l}\text { Chat } \\
\text { Index }\end{array}$ & $\begin{array}{l}\text { Time } \\
\text { Start } \\
\text { Typing }\end{array}$ & $\begin{array}{l}\text { Time of } \\
\text { Posting }\end{array}$ & Author & Content & Refers to \\
\hline \multirow[t]{2}{*}{818} & $19: 51: 11$ & 19:52:19 & qwertyuiop & what about: $f(n)=2 n-1$ where $n$ is side length & Message No. 772 \\
\hline & & $19: 52: 28$ & 137 & $\begin{array}{l}137 \text { changes the layout of the last straight } \\
\text { line by making it a dashed line. }\end{array}$ & \\
\hline 819 & 19:52:55 & 19:53:03 & 137 & I don't think that works. & Message No. 818 \\
\hline 820 & 19:53:07 & 19:53:18 & 137 & Howbout just $n(n+1) / 2$ & \\
\hline 821 & 19:53:37 & 19:53:41 & Jason & for \# sticks? & \\
\hline 822 & $19: 53: 38$ & $19: 53: 48$ & qwertyuiop & that's number of sides for one set & Message No. 820 \\
\hline 823 & $19: 53: 50$ & 19:53:51 & qwertyuiop & $?$ & \\
\hline 824 & $19: 53: 57$ & 19:53:59 & Jason & oh ok nvm & \\
\hline 825 & $19: 54: 26$ & $19: 54: 29$ & 137 & Ya. & Message No. 822 \\
\hline
\end{tabular}

(Hanks, 2000, p. 8.). These excerpts show that mathematical terms are inherently indexical. Establishing a shared understanding of such indexical terms require collaborators to establish a reciprocity of perspectives towards the reasoning practices displayed/embodied in the organization of the texts and inscriptions in the shared scene (Zemel \& Cakir, 2009).

${ }^{9}$ A brief administrative episode including the facilitator took place between excerpts 4 and 5 , which is omitted in an effort to keep the focus of our analysis on problem solving. 


\begin{tabular}{|c|c|c|c|c|c|}
\hline 826 & $19: 54: 36$ & $19: 54: 58$ & qwertyuiop & then $x 3$ is $3(n(n+1) / 2)$ & Message No. 820 \\
\hline 827 & 19:55:04 & 19:55:07 & qwertyuiop & simplified to... & Message No. 826 \\
\hline 828 & 19:55:11 & $19: 55: 37$ & qwertyuiop & $(n(n+1) 1.5$ & \\
\hline \multirow[t]{2}{*}{829} & $19: 55: 34$ & $19: 55: 44$ & 137 & $\begin{array}{l}\text { On second thought, shouldn't we use } n(n-1) \\
\text { for these: }\end{array}$ & Message No. 826 \\
\hline & & $\begin{array}{l}19: 55: 50 \\
- \\
19: 55: 55\end{array}$ & 137 & $\begin{array}{l}137 \text { changes the color of two dashed lines into } \\
\text { orange (see Figure } 13 \text { below) }\end{array}$ & \\
\hline 830 & 19:55:31 & 19:55:55 & Nan & $\begin{array}{l}\text { just a kind reminder: Jason mentioned that he } \\
\text { needs to leave at } 7 p \text { central time sharp }\end{array}$ & \\
\hline
\end{tabular}

137 rejects Qwertyuiop's proposal in line 819 and then makes a counter proposal in the next line. As we saw in Excerpt 3, the sequence of numbers resulting from Qwertyuiop's counting method was previously associated with a math artifact called triangular numbers by 137 . The counter proposal includes the same expression 137 provided earlier when he gave a definition of triangular numbers as "integers that can be represented as $n(n+1) / 2$ " (see line 774). Jason joins the discussion in line 821 by asking if the proposed formula is for the number (“\#”) of sticks. Although Jason does not specify which object (e.g., the whole hexagon) he is associating the formula with, his posting can be read as an attempt to solicit further elaboration with regards to what the recently proposed formulas are about.

Qwertyuiop's posting in the next line states that the object indexed by the deictic term "that" corresponds to the "number of sides for one set". Note that Qwertyuiop's message is explicitly linked to 137's counterproposal in line 820, so the deictic term "that" can be read as a reference to the expression " $n(n+1) / 2$ " included in 137's posting. Moreover, the message sequentially follows Jason's question. Hence, Qwertyuiop seems to be responding to Jason's query by pointing out which object the recently proposed formulas are about. The question mark Qwertyuiop posts in the next line mitigates his previous statement into a question. This can be read as a move to solicit the remaining member's (i.e. 137) assessment of the association Qwertyuiop has just offered. By making his reading of 137's formula explicit, Qwertyuiop also indicates that he concurs with the alternative expression proposed by his peer. Jason's next posting in line 824 indicates that he is now following his peers' reasoning, which comes just before 137's confirmation linked to Qwertyuiop's claim in 822. Therefore, at this point it seems to be evident for all members in the group that the algebraic expression $n(n+1) / 2$ is associated with one of the "collinear sets of sticks" within a triangular section.

In line 826, Qwertyuiop posts a message linked back to 137's proposal in 820 . The use of "then" at the beginning suggests that this message is a consequence or follow up of the message he is referring to. " $x 3$ " can be read as a reference to multiplication by 3 , where the remaining part of the message provides the expression yielded by this operation. In other words, Qwertyuiop seems to be proposing the next step in the computation, given the expression for the number of sticks for a single "set". In the next two lines he further simplifies this expression by evaluating $3 / 2$ to 1.5 .

In line 829,137 posts a message phrased as a question. The posting begins with "on second thought" which indicates that the author is about to change a position he took prior with respect to the matter at hand. The rest of the statement is phrased as a question and it is addressed to the whole team as indicated by the use of the first person plural pronoun "we". The question part associates the expression "n(n-1)" 
with the deictic term "these" which is yet to be specified ${ }^{10}$. The posting ends with ":" which projects that more content will likely follow this message subsequently. Next, 137 begins to act on the whiteboard by changing the color of two horizontal lines from green to orange (see Figure 14). The temporal unfolding of these actions suggests that the sticks highlighted in orange are somehow associated with the expression $\mathrm{n}(\mathrm{n}-1)$. In other words, 137's recent actions can be seen as a move for adjusting the index values in the generalized formula.
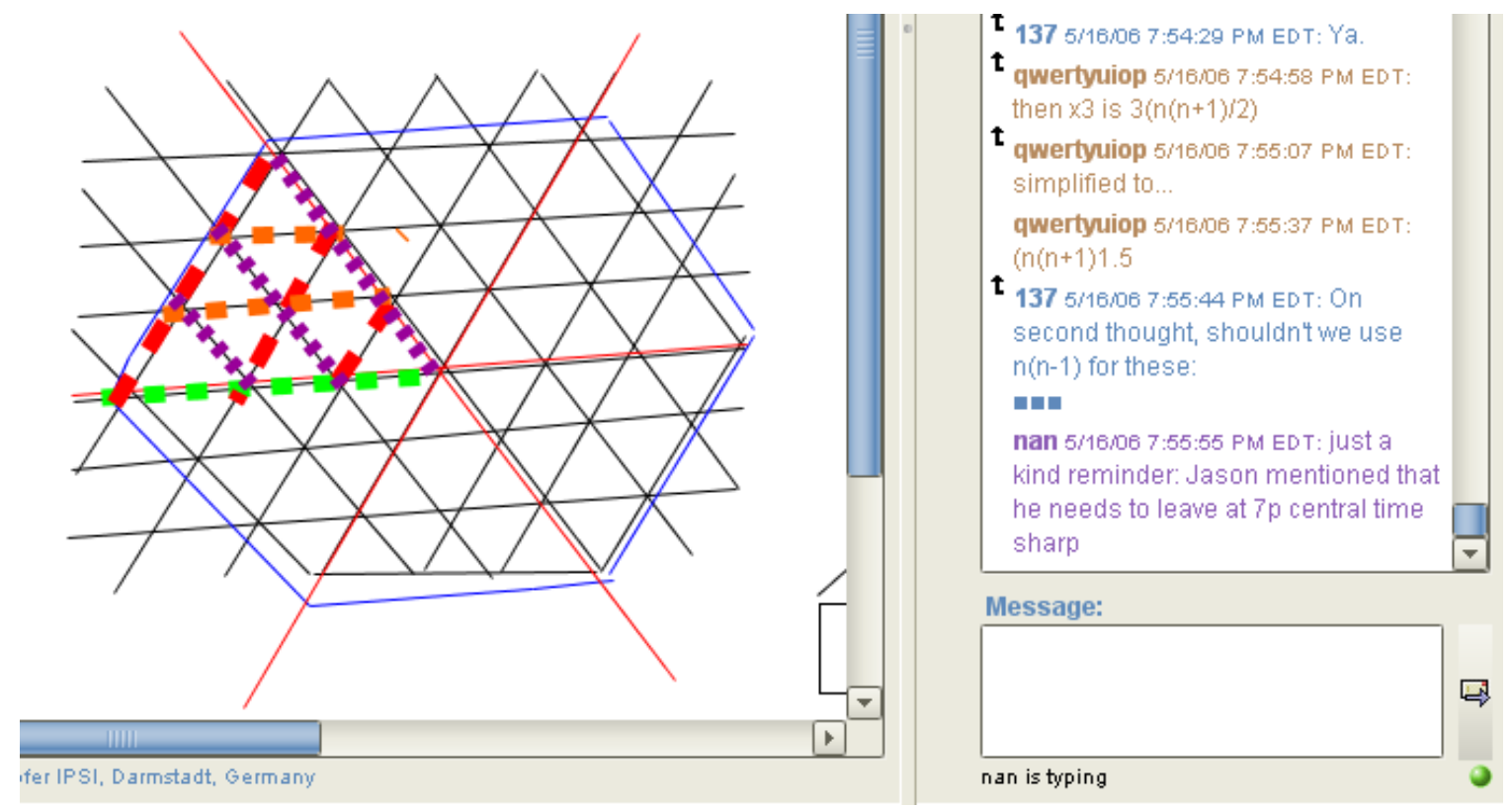

Figure 14: 137 highlights 2 horizontal lines in orange following his proposal at 7:55:44 (line 829).

In this episode, the team achieves an important transition from a geometrically motivated counting procedure applied on "one of the collinear sets" to a symbolic formula generalizing the procedure to a set of any given sidelength. The generality is achieved through one member's noticing that the sequence of numbers derived from the counting procedure corresponds to "triangular numbers", which seems to be a familiar concept at least for the member who proposed it. The formula that was provided as part of the definition of triangular numbers is then applied to the relevant portion of the pattern at hand to achieve the transition from geometric to algebraic mode of reasoning, mediated by the narrative concept of "triangular numbers".

Excerpt 6: Co-reflection on what the team has achieved so far

\begin{tabular}{|l|l|l|l|l|l|}
\hline $\begin{array}{l}\text { Chat } \\
\text { Index }\end{array}$ & $\begin{array}{l}\text { Time } \\
\text { Start } \\
\text { Typing }\end{array}$ & $\begin{array}{l}\text { Time of } \\
\text { Posting }\end{array}$ & Author & Content & Refers to \\
\hline 841 & $19: 58: 23$ & $19: 58: 25$ & qwertyuiop & Back to this? & Message No. 829 \\
\hline 842 & $19: 58: 32$ & $19: 58: 34$ & 137 & Ya & Message No. 829 \\
\hline 843 & $19: 58: 39$ & $19: 58: 49$ & qwertyuiop & why not n(n-1)? & \\
\hline 844 & $19: 58: 39$ & $19: 58: 50$ & Jason & you guys pretty much have the formula for this & \\
\hline
\end{tabular}

\footnotetext{
${ }^{10}$ See footnote to line 746 on the use of "these" and "those". The consistency of the usage of these terms for forward and backward references from the narrative chat to the graphical whiteboard suggests an established syntax of the relationships bridging those interaction spaces within the temporal structure of the multi-modal discourse.
} 


\begin{tabular}{|l|l|l|l|l|l|}
\hline 845 & $19: 58: 57$ & $19: 59: 28$ & qwertyuiop & $\begin{array}{l}\text { hexagon problem... } \\
\text { We almost have it for the triangle. I don't know } \\
\text { about the hexagon. }\end{array}$ & Message No. 844 \\
\hline 846 & $19: 59: 35$ & $19: 59: 50$ & Jason & $\begin{array}{l}\text { well that's just multiplied by a certain number } \\
\text { for a hexagon, provided that it is regular }\end{array}$ & Message No. 845 \\
\hline 847 & $19: 59: 58$ & $20: 00: 14$ & qwertyuiop & $\begin{array}{l}\text { but the sides of the triangles making up the } \\
\text { hexagon overlap }\end{array}$ & Message No. 846 \\
\hline 848 & $19: 59: 52$ & $20: 00: 18$ & Jason & $\begin{array}{l}\text { well i have to leave now; sorry for not } \\
\text { participating as much as i wanted to, it's a pretty } \\
\text { busy night for me with school and } \\
\text { extracurricular stuff }\end{array}$ & \\
\hline
\end{tabular}

At the end of excerpt 5 an administrative discussion was initiated by the facilitator about Jason's departure from the chat session ${ }^{11}$. Some of this exchange is left out since it involved a brief chat about the schedule of the next session. However, while Jason was saying farewell to his peers, an exchange related to the task at hand occurred which is captured in Excerpt 6. This episode begins with Qwertyuiop's attempt to reinitiate the problem-solving work by making a reference to an older message posted in line 829 by 137. Following 137's acknowledgement in line 842, Qwertyuiop posts a question linked to line 829 which indicates that he is oriented to the expression 137 proposed in that message.

About a second later, Jason posts a message stating that the formula for the hexagon problem is pretty much done. Jason's use of the phrase "you guys" ascribes this achievement to the remaining members of the team. In line 845, Qwertyuiop posts a message explicitly linked to Jason's last comment. The first sentence "We almost have it for the triangle" provides an alternative account of what has been achieved so far. In his second sentence, Qwertyuiop declares that he does not know about the hexagon yet. Hence, these postings make it evident how Qwertyuiop is treating what the team has accomplished so far.

In line 846, Jason posts a message linked to Qwertyuiop's latest remark. In his response Jason states that getting the formula for the hexagon requires a simple multiplicative step provided that the hexagon is regular. Qwertyuiop's response (as indicated by the referential arrow) follows next, where he brings in how the issue of overlap will play out when they move from the large triangles to the whole hexagon. This is followed by Jason's exiting remark where he apologizes for not being able to participate as much as he wanted.

In this excerpt, team members explicitly commented on how they characterize their collective achievement. In other words, these postings can be read as a joint reflection on what has been done so far. Another interesting aspect of this short exchange is the apparent shift in the positions with respect to the issue of overlapping sticks in the counting procedure. Jason was the person who raised the issue of overlap for the first time in excerpt 3, yet his most recent characterization of the team's work seems to dismiss overlap as a relevant matter. Surprisingly, Qwertyuiop, who was the person previously critiqued by Jason for possibly ignoring the issue of overlapping sticks, explains now why it is a relevant matter that needs to be attended to, before the number of sticks in one triangle is multiplied by a certain number as Jason suggested in 846. In excerpt 3, Qwertyuiop argued that overlaps would not be an issue in his counting work, but that assertion seems to be applied only to the triangular section he was oriented to at that time. His most recent posting displays his awareness with regards to when the overlapping sticks will become an issue, i.e. when they move from the triangular partition to the whole hexagon. These remarks

\footnotetext{
${ }^{11}$ The session was scheduled to end at $7 \mathrm{pm}$, yet the students were allowed to continue if they wished to do so. In this case Jason informed the facilitators in advance that he had to leave at $7 \mathrm{pm}$ Central (the log is displayed in US Eastern time).
} 
also specify what has not been accomplished yet, and hence suggest the team to find a way to address overlaps as an issue to consider next.

Excerpt 7: Overcoming the problem of overlapping sticks

\begin{tabular}{|c|c|c|c|c|c|}
\hline $\begin{array}{l}\text { Chat } \\
\text { Index }\end{array}$ & $\begin{array}{l}\text { Time } \\
\text { Start } \\
\text { Typing }\end{array}$ & $\begin{array}{l}\text { Time of } \\
\text { Posting }\end{array}$ & Author & Content & Refers to \\
\hline 853 & & 20:01:07 & & Jason leaves the room & \\
\hline 854 & 20:01:19 & 20:01:31 & 137 & $\begin{array}{l}\text { Anyways, if we multiply the orange by } 3 \text {, we } \\
\text { get the: }\end{array}$ & \\
\hline \multirow[t]{2}{*}{855} & 20:01:14 & 20:01:34 & Nan & $\begin{array}{l}\text { do two of you want to continue working for a } \\
\text { bit or stop here? }\end{array}$ & \\
\hline & & $\begin{array}{l}\text { 20:01:42 } \\
- \\
20: 01: 48\end{array}$ & 137 & $\begin{array}{l}137 \text { begins to add blue lines on top of the } \\
\text { triangular grid }\end{array}$ & \\
\hline 856 & 20:01:40 & 20:01:44 & Nan & i guess that's the answer & Message No. 854 \\
\hline \multirow[t]{2}{*}{857} & 20:01:47 & 20:01:48 & Nan & go ahead & \\
\hline & & $\begin{array}{l}20: 01: 49 \\
- \\
20: 01: 53\end{array}$ & 137 & $\begin{array}{l}137 \text { continues to add blue lines. The resulting } \\
\text { shape is displayed in Figure } 15\end{array}$ & \\
\hline 858 & 20:01:57 & 20:02:14 & 137 & So then we add $12 n$ for: & \\
\hline \multirow[t]{2}{*}{859} & 20:01:28 & 20:02:15 & qwertyuiop & $\begin{array}{l}\text { actually, this doesn't complicate it that much. } \\
\text { The overlaps can be accounted for with "-6n" }\end{array}$ & Message No. 847 \\
\hline & & $\begin{array}{l}20: 02: 32 \\
- \\
20: 02: 52\end{array}$ & 137 & $\begin{array}{l}137 \text { adds pink contours to the shared } \\
\text { drawing, The resulting shape is displayed in } \\
\text { Figure } 16\end{array}$ & \\
\hline 860 & 20:02:54 & 20:02:55 & 137 & Oh. & Message No. 859 \\
\hline 861 & 20:02:56 & 20:03:07 & 137 & I like addition more than subtraction. & \\
\hline
\end{tabular}

Excerpt 7 follows Jason's departure ${ }^{12}$. In line 854,137 re-initiates the problem-solving work by proposing to multiply by 3 what is indexed by "the orange". Figure 15 shows the state of the shared drawing at the moment, where there are two dashed orange lines covering a portion of the hexagon. The remaining part of the message announces the outcome of the suggested operation, but no result is provided yet. The message ends with a colon ":" indicating that more content is about to follow subsequently. Next, 137 performs a series of drawing actions where he highlights a set of sticks on the triangular grid with blue lines (see Figure 16). These actions are done within a section of the shared drawing that has been empty. Based on the way these actions sequentially unfold and the way the drawing was set up in chat, one can read these actions as the visual outcome of the operation described in text in line 854. In short, multiplying the number of orange dashed lines by 3 seems to yield the number of sticks highlighted in blue, which is an elaborate mathematical move spanning across textual and graphical modalities.

\footnotetext{
${ }^{12}$ The facilitator opens the possibility to end the session in line 855 . The facilitator takes the sustained orientation of the remaining team members to the problem as an affirmative answer and lets the team continue their work.
} 


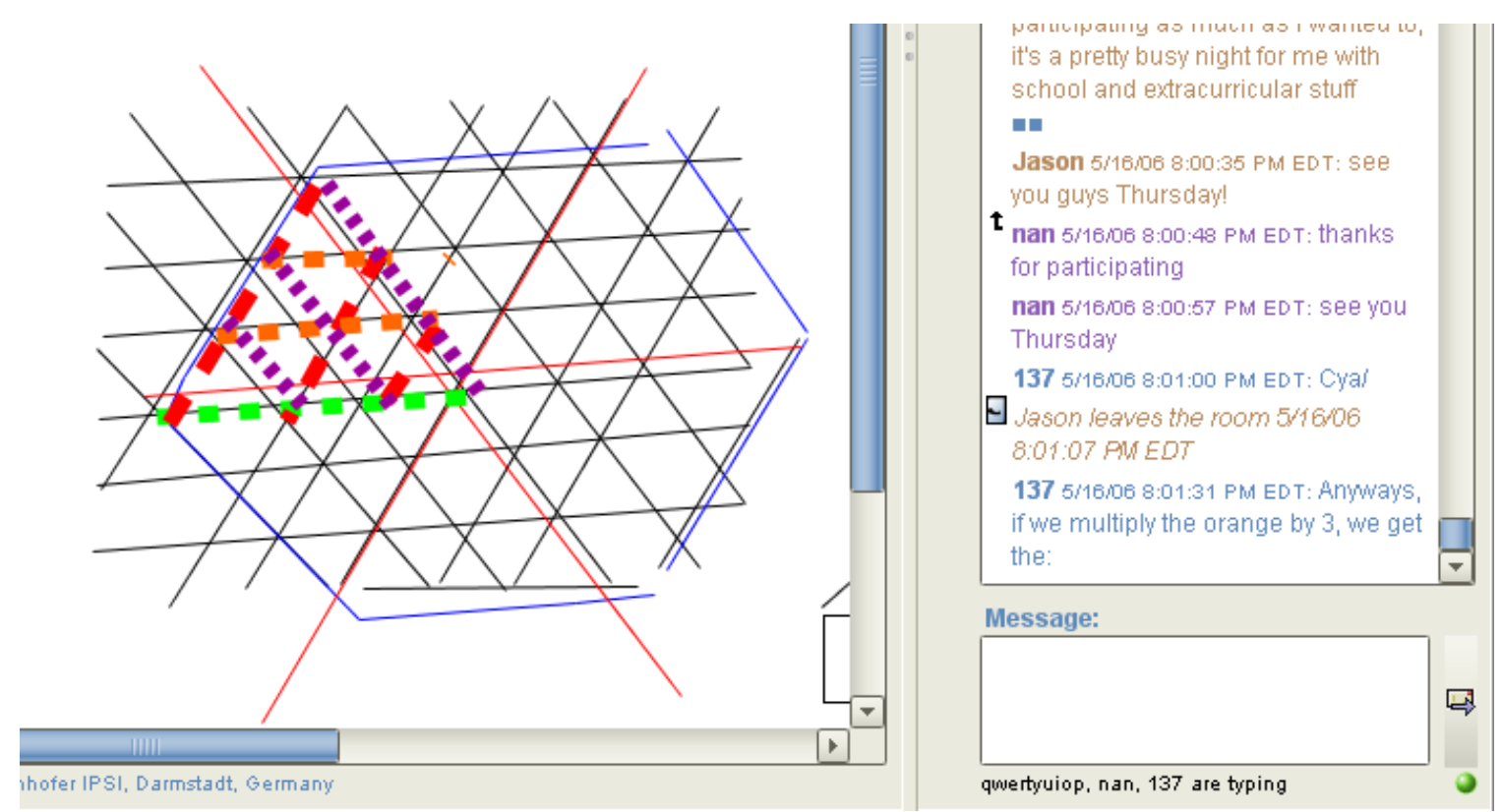

Figure 15: The state of the whiteboard when 137 began his exposition at 8:01:31 (line 854)

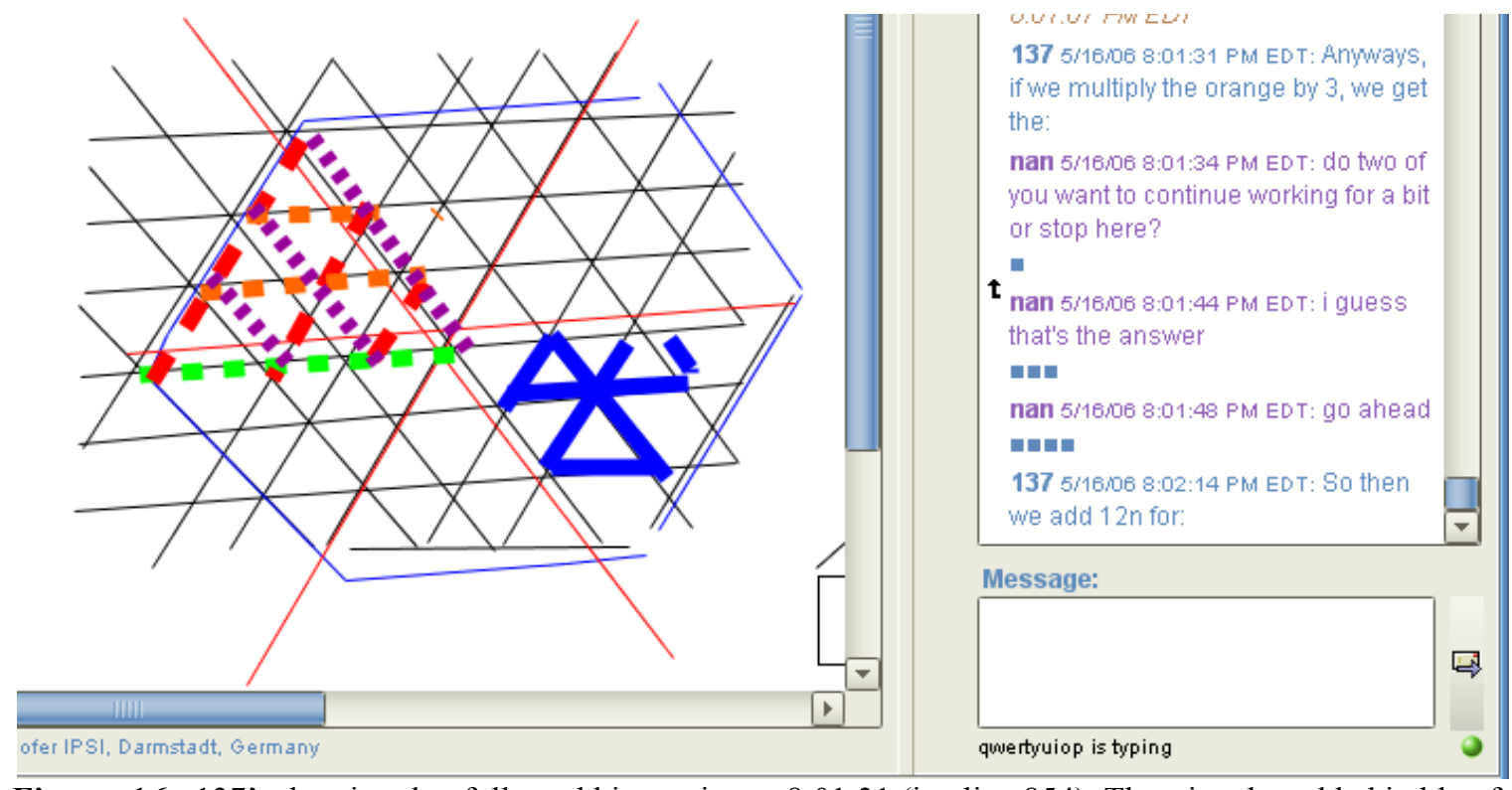

Figure 16: 137's drawing that followed his posting at 8:01:31 (i.e. line 854). The triangles added in blue follow the chat posting that proposes the multiplication of what is marked with orange by 3 .

137 posts another message in line 858 which announces adding " $12 n$ " as the next step in his ongoing exposition. The message ends with "for:" which is consistent with his prior use of the colon to project that more elaboration will follow, possibly in the other interaction space. Next, 137 begins to add pink lines to the shared drawing, which covers the boundaries and the diagonals of the hexagonal array (see Figure 17). The sequential continuity of 137's actions suggests that the lines marked with pink provide a geometric realization of what is indexed by the symbolic expression " $12 n$ " on the particular instance represented by the shared drawing. 


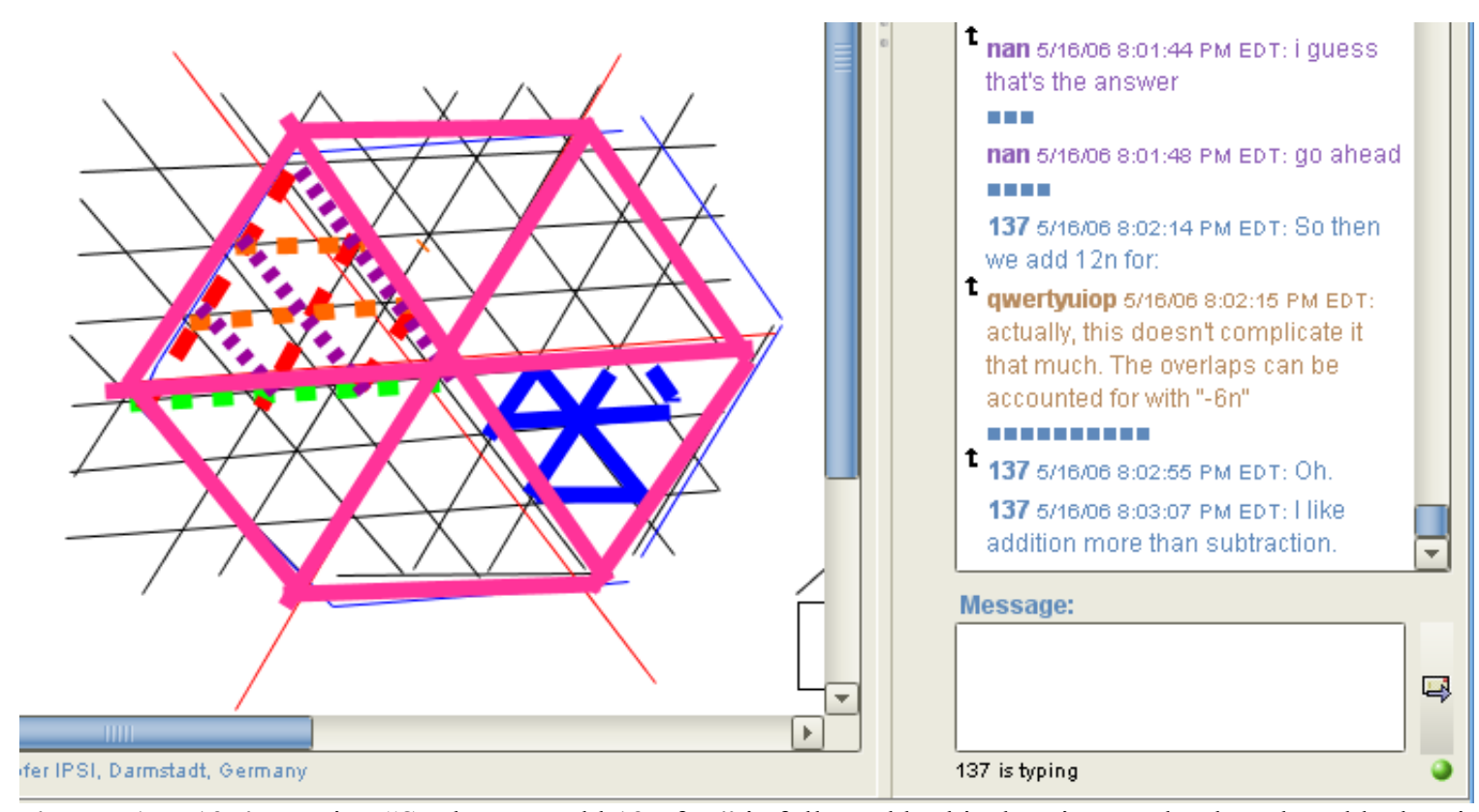

Figure 17: 137's posting "So then we add 12n for:" is followed by his drawing work where he adds the pink lines. Again the temporal sequencing suggests that the pink lines show visually which sticks will be covered when the proposed computation is performed (i.e., "adding $12 \mathrm{n}$ ")

While 137 was composing his message, Qwertyuiop was busy typing the message that will appear in line 859. The message appears 1 second after 137's posting and just before he begins adding the pink lines. Hence, the temporal unfolding of actions suggests that these two messages were produced in parallel. In this posting Qwertyuiop makes a reference to an older message where he mentioned the problem of overlapping sticks among the 6 triangular regions. The current message announces that this may not be a big complication. The next sentence in the same post states that the overlaps can be accounted for with the expression “-6n". 137's response (as suggested by his use of the explicit reference) to Qwertyuiop's proposal comes after he is done with marking the pink lines on the whiteboard. The "oh" in line 861 makes 137's noticing of Qwertyuiop's proposal. In his next posting, 137 states that he prefers addition rather than subtraction. The contrast made between addition and subtraction suggests that 137 is treating his and Qwertyuiop's methods as distinct but related approaches to the task at hand.

What 137 is referring to as an "additive" approach can be observed through his prior actions distributed across both interaction spaces. 137's approach begins with a method to cover a specific portion of one of the six partitions of the hexagon. This is referred as "multiplying the orange by three" and the outcome of this operation is marked in blue. In other words, the orange lines seem to be used as a way to index a single side of a total of $1+2=3$ triangles (or $n(n-1) / 2$ in general) inside one of the 6 partitions. Hence, multiplying this value by 3 covers the 3 blue triangles enclosed in a partition. Moreover, none of these triangles share a stick with the diagonals and the boundary of the hexagon, so the sticks highlighted in pink are added to cover the missing sticks. In short, 137's reasoning for the additive approach is evidenced in his drawing actions as well as in the way he coordinated his chat postings with the drawings.

The other approach referred to as "subtraction" by 137 has been discussed by the team for a while. This approach starts with counting the sticks for one of the six partitions of the hexagon. A partition is further split into "3 collinear sets" of sticks that do not "overlap" with each other. The number of sticks covered by a single set turned out to be equivalent to a "triangular number". Nevertheless, since this approach covers all the sticks forming a partition and partitions share a boundary with their neighbors, when this value is multiplied by 6 to cover the whole hexagon, the sticks at the boundaries (i.e., at the diagonals) would be counted twice. This is referred to by the team as the overlap problem. Qwertyuiop's latest 
proposal provides the expression that needs to be subtracted from the general formula to make sure all sticks at the internal boundaries are counted exactly once. In contrast, the additive approach does not need subtraction since it splits the shape in such a way that each stick is counted exactly once.

The main point we would like to make about this excerpt is that 137's approach takes the previously demonstrated approaches and their critiques as resources. He offers a new approach informed by previous discussion in an effort to address the practical issues witnessed (e.g., overlaps, adjusting the index in the expression for triangular numbers, etc.). Hence, 137's additive approach is firmly situated within the ongoing discussion. In other words, 137's reasoning has been socially shaped; it is not a pure cognitive accomplishment of an individual mind working in isolation from others.

Excerpt 8: Derivation of the formula for the number of sticks

\begin{tabular}{|c|c|c|c|c|c|}
\hline $\begin{array}{l}\text { Chat } \\
\text { Index }\end{array}$ & $\begin{array}{l}\text { Time } \\
\text { Start } \\
\text { Typing }\end{array}$ & $\begin{array}{l}\text { Time of } \\
\text { Posting }\end{array}$ & Author & Content & Refers to \\
\hline 862 & 20:03:11 & 20:03:16 & Qwertyuiop & do you see why that works & Message No. 859 \\
\hline 863 & 20:03:18 & 20:03:18 & Qwertyuiop & $?$ & \\
\hline 864 & 20:03:12 & $20: 03: 29$ & 137 & So: $9 n(n+1)-6 n$. & \\
\hline 865 & 20:03:41 & 20:03:45 & Qwertyuiop & 9, not $3 ?$ & \\
\hline 866 & 20:04:13 & 20:04:14 & 137 & $?$ & Message No. 865 \\
\hline 867 & 20:04:18 & $20: 04: 35$ & Qwertyuiop & you have "9n(n..." & \\
\hline 868 & $20: 04: 37$ & $20: 04: 47$ & Qwertyuiop & not "3n(n..."? & \\
\hline 869 & 20:04:51 & 20:05:00 & 137 & But we need to multiply by 6 then divide by 2 & Message No. 868 \\
\hline 870 & 20:05:10 & $20: 05: 22$ & Qwertyuiop & $x 6$ and $/ 2$ for what? & Message No. 869 \\
\hline 871 & 20:05:44 & 20:05:47 & 137 & FOr each triangle & \\
\hline 872 & 20:05:48 & 20:06:02 & 137 & and $/ 2$ because it's part of the equation. & \\
\hline 873 & 20:06:03 & 20:06:06 & 137 & of $n(n+1) / 2$ & \\
\hline 874 & 20:05:36 & 20:06:20 & Qwertyuiop & $\begin{array}{l}\text { it's } \times 3 \text { for the } 3 \text { colinear sets, then } \times 6 \text { for } 6 \\
\text { triangles in a hexagon... where's the } 9 \text { and } 2 \text { ? }\end{array}$ & \\
\hline 875 & $20: 06: 28$ & 20:06:28 & Qwertyuiop & $\mathrm{Oh}$ & Message No. 872 \\
\hline 876 & 20:06:35 & 20:06:38 & 137 & So $18 / 2$ & \\
\hline 877 & 20:06:42 & 20:06:50 & 137 & A.K.A. 9 & \\
\hline 878 & 20:06:48 & 20:07:08 & Qwertyuiop & $(\mathrm{n}(\mathrm{n}+1) / 2) \times 3 \times 6$ & Message No. 873 \\
\hline 879 & 20:07:14 & 20:07:15 & 137 & Yeah. & \\
\hline 880 & 20:07:20 & 20:07:27 & Qwertyuiop & Which can be simplified... & \\
\hline 881 & 20:07:42 & 20:07:46 & 137 & To $9 n(n+1)$ & Message No. 880 \\
\hline 882 & 20:08:01 & 20:08:04 & Qwertyuiop & that's it? & Message No. 881 \\
\hline 883 & 20:08:10 & 20:08:12 & 137 & $-6 n$. & \\
\hline 884 & 20:08:17 & 20:08:24 & 137 & So $9 n(n+1)-6 n$ & \\
\hline 885 & $20: 08: 20$ & 20:08:34 & Qwertyuiop & i'll put it with the other formulas... & \\
\hline
\end{tabular}

Excerpt 8 immediately follows the prior one. It begins with Qwertyuiop's question addressed to 137, which asks if he could see why subtracting $6 \mathrm{n}$ would work. In the meantime, 137 seems to be busy typing 
the message that will appear in line 864. The use of "So" suggests that this message is stated as a consequence of what has been discussed so far. The colon is followed by the formula " $9 n(n+1)-6 n$ ", which involves the term " $-6 n$ " in it. By using the term " $-6 n$ ", 137 makes his orientation to Qwertyuiop's proposal explicit. Moreover, the sequential build up suggests that the proposed expression stands for the formula for the number of sticks for the hexagonal array. In these ways - through the details of its contextual situating - the symbolic expression is tied to the on-going discourse, including the graphical features.

Qwertyuiop's next posting in line 864 problematizes the appearance of 9 in the proposed formula and asks if 3 should have appeared there instead. Next, 137 posts a question mark linked to Qwertyuiop's question, which can be read as a request for more elaboration. Qwertyuiop elaborates in the next two lines by posting the part of the formula that is problematic for him and then by suggesting a repair for that part. His elaboration ends with a question mark that can be seen as an attempt to solicit his peer's feedback. 137's reply in line 869 states that the steps of the computation should also include multiplication by 6 and division by 2. In response Qwertyuiop asks for what part of the pattern those operations need to be done. 137's reply spans 3 lines, where he first states "for each triangle" and then mentions that "/2" comes from the equation $n(n+1) / 2$. Hence the sequential organization of these messages suggest that 137 associates multiplication by 6 with the triangles (i.e., the larger triangular partitions) and "/2" with the equation for triangular numbers.

In the meantime, Qwertyuiop has been typing what will appear in line 874 . The first sentence associates each multiplication operation with a specific section of the hexagonal pattern, namely " $x 3$ " for the 3 "collinear sets" within a triangular partition and " $x 6$ " for the 6 triangular partitions of the hexagon. The next sentence in that posting problematizes again the appearance of 9 and 2 in the steps of the calculation. Eight seconds later, Qwertyuiop posts “oh" in response to 137's remark about the equation in line 872, which indicates that the referenced message has led him to notice something new. This is followed by 137's demonstration of the derivation of 9 from the numbers previously mentioned. Meanwhile, Qwertyuiop is composing an expression that brings all the items they have just talked about together in symbolic form, which appears in line 878 in response to line 873 where 137 reminded him about the equation $n(n+1) / 2.137$ expresses his agreement in the next line. Next, they simplify the expression and add" $-6 n$ " to derive the final formula for the number of sticks.

In short, the episode following 137's proposal shows that Qwertyuiop had trouble understanding how 137 derived the formula he reported in line 864.137 seems to have gone ahead with putting together all the different pieces of the problem that have been discussed so far to produce the final formula. Note that the additive approach 137 was describing earlier included a step summarizing the pink boundary as $12 \mathrm{n}$, which also includes the diagonals causing the overlap issue. The commonality between the two lines of reasoning may have informed 137's quick recognition of the algebraic implication of Qwertyuiop's subtraction move as an alternative to his approach.

Qwertyuiop's problematizations of some of the terms that appear in the proposed formula have led 137 to reveal more details of his algebraic derivation. This exchange has revealed how each algebraic move is based on the corresponding concept the team had developed earlier (e.g., $n(n+1) / 2$ sticks to cover a collinear set, multiply by 3 to cover 3 collinear sets making up a triangular partition, multiply by 6 to cover the hexagon, subtract $6 \mathrm{n}$ to remove those sticks at the internal boundaries that are counted twice). 137's contributions in this and the previous excerpts demonstrate that he can competently associate the narrative descriptions and visual representations with symbolic formulas. Qwertyuiop's initial trouble and its resolution in the last excerpt provided us further evidence with regards to how participants made use of the narrative/geometric resources to co-construct a generalized symbolic formula addressing the problem at hand. In short, the team members complemented each other's skills as they incorporated geometric and algebraic insights proposed by different members into a solution for the task at hand during the course of their one hour long chat session. 


\section{Discussion}

In this section we discuss the findings of our case study regarding the affordances of a multimodal CSCL environment for joint mathematical meaning making online and the interactional organization of mathematics discourse.

\section{Visibility of the Production Process}

Our first observation is related to the mathematical affordances of the drawing area. As we have seen in Excerpts 1, 2, 4, and 7, the construction of most shared diagrams includes multiple steps (e.g., addition of several lines). Moreover, the object-oriented design of the whiteboard allows users to re-organize its content by adding new objects and by moving, annotating, deleting and reproducing existing ones. Hence, the sequencing of drawing actions that produce and/or modify these diagrams is available for other members to observe. In other words, the whiteboard affords an animated evolution of the shared space, which makes the reasoning process visually manifest in drawing actions available for other members to observe. For instance, the sequence of drawing actions that led to the drawing displayed in Figure 13 (Excerpt 4) allowed the team members to locate what was indexed by the term "set of 3 collinear sides." The drawing also served as a visual proof for the argument that those three sets do not share any sticks (i.e., they do not overlap). Finally, Figures 16 and 17 show cases where a textually described algebraic operation was subsequently animated on the whiteboard. Such demonstrable tweaks make the mathematical details of the construction work visible and relevant to observers, and hence serve as a vital resource for joint mathematical sense making.

\section{Persistent Presence of Contributions}

In the VMT online environment, contributions have a persistent presence that allows participants to revisit a prior posting or reorganize a shared drawing to orient themselves to shared artifacts in new ways. One important consequence of persistence is illustrated by Qwertyuiop in Excerpts 4 and 5 (lines 803 and 818) and by 137 in Excerpt 3 (line 776), where they used the explicit referencing tool to point to a previous chat posting in an effort to re-initiate a past topic or thread. When combined with the referential arrows, the persistent availability of the chat messages affords re-initiation of past conversations and the management of multiple threads (e.g., the discussion on a missing stick and the formula for triangular numbers that unfolded in parallel in Excerpts 2 and 3 illustrates how users manage multiple threads).

One important consequence of quasi-synchronous interactions mediated by a persistent display of text messages is that participants are not subjected to the same set of physical constraints underlying the turntaking apparatus associated with talk in face-to-face settings. In natural conversations, speakers take turns due to the practical intelligibility issues involved with overlapping speech. In contrast, the persistent availability of the text messages affords simultaneous production of contributions, and hence provides more possibilities for participation. This may introduce intelligibility issues referred to as chat confusion (Fuks, Pimentel \& de Lucena, 2006) or phantom adjacency pairs (Garcia \& Jacobs, 1998), when simultaneously produced messages can be mistakenly treated in relation to each other. However, as we have seen in the excerpts analyzed above, participants routinely provide enough specificity to their contributions (e.g., by using the referential tool or specific tokens) and orient to the temporal/linear order in which messages appear on the screen to avoid such issues of intelligibility. Finally, when coupled with resources such as the explicit referencing tool and repetition of specific terms (e.g., "sidelength"), the persistency of chat messages also allows participants to make a previous discussion relevant to the current discussion. For instance, in line 818 in Excerpt 5, Qwertyuiop re-oriented the current discussion to the issue of devising a formula for the sequence of numbers that was stated back in line 772 by using the explicit referencing tool. Likewise, in line 841 in Excerpt 6 Qwertyuiop proposed that the team re-initiate a discussion on a point stated 13 lines above with his message "go back to this" coupled with an explicit referential link. 
The possibility of engaging activities across multiple threads spanning both chat and whiteboard spaces is an important affordance of online environments like VMT due to the opportunities it brings in for more people to contribute to the ongoing discussion. For instance, in Excerpt 4 we have seen that 137 was engaged in two simultaneous threads where (a) he drew a line segment that was potentially ignored by the method of computation described by Qwertyuiop, and (b) he contributed to the simultaneously unfolding discussion about characterizing the pattern implicated by the numbers offered by Qwertyuiop as triangular numbers. Although the management of multiple threads across spaces can create confusion, the resolution of ambiguities and the intertwining of perspectives can lead to germination/fertilization of mathematical ideas across threads. This point is well demonstrated by how the aforementioned threads led to Qwertyuiop's visual proof, which (a) located visually what the term "3 sets of collinear lines" meant, (b) established that the sets do not overlap with each other, and (c) highlighted the association between the cardinality of a single set and a triangular number.

Finally, there is a subtle but important difference between the chat and whiteboard features in terms of the degree of persistence of their contents. As a session progresses chat postings gradually scroll away, but whiteboard drawings stay on the whiteboard until they are erased. For instance, in all the excerpts we have seen above, the particular illustration of the hexagonal pattern continued to serve as an interactional resource as team members illustrated and offered different ideas. Several chat postings presume the availability of such a persistent resource on display so that others can make sense of the contribution (e.g., indexical terms such as "the orange", "3 sets", etc.). Such persistently available artifacts provided the background against which new contributions were interpreted and made sense of.

\section{Methods for Referencing Relevant Artifacts in the Shared Visual Field}

Bringing relevant mathematical artifacts to other members' attention requires a coordinated sequence of actions performed in both the chat and whiteboard spaces (Stahl et al., 2011). In the excerpts above we have observed several referential methods enacted by participants to bring relevant graphical objects on the whiteboard to other group members' attention. In Excerpt 1, 137 marked the drawing with a different color to identify what he thought collinear sides meant in reference to the shared drawing. Qwertyuiop also used the same approach when he highlighted the collinear sides in the shared drawing with different colors in Excerpts 1 and 3. Color coding was another method used by members to draw others' attention to specific parts of the drawing (e.g., "the orange", "the green times 3"). Finally, members used the explicit referencing tool to support their textual descriptions. For instance, Qwertyuiop used the explicit referencing tool in Excerpts 2 and 4 to direct his teammates' attention to the relevant section of the hexagon where he was performing his counting work. In all these cases, chat messages included either an explicit reference or a deictic term such as "this", "that", or "the green", which are designed to inform other members of the group that they need to attend to some features beyond the textual statement itself to make sense of the chat message.

These referential mechanisms play a key role in directing other members' attention to features of the shared visual field in particular ways. This kind of deictic usage isolates components of the shared drawing and constitutes them as relevant objects to be attended to for the purposes at hand. Hence, such referential work establishes a fundamental relationship between the narrative and mathematical terminology used in text chat and the animated graphical constructions produced on the whiteboard. The shared sense of the textual terms and the inscriptions co-evolve through the referential linkages established as the interaction sequentially unfolds in the dual-interaction space.

Deictic uses of text messages and drawings presume the availability of a shared indexical ground (Hanks, 1992) where the referential action can be seen as the figure oriented towards some part of the shared background. In other words, referential moves are not performed in isolation; they rely on a part/whole relationship between the referential action (i.e., figure) and a shared visual ground. For example, the color markings of collinear lines in Excerpt 4 worked as a referential action, because they were performed on 
top of an existing graphical artifact, namely the triangular grid. Even the design of the explicit referential tool, which attaches a semi-transparent green rectangle to a chat message, reflects this visual relationship between the figure (i.e., the green rectangle) and the background, which guides other members' attention to a particular location in the shared visual field. As virtual teams collaboratively explore their problem and co-construct shared artifacts, they collectively constitute a shared problem space with increasing complexity (Sarmeinto \& Stahl, 2008). By enacting referential practices, participants isolate features of the shared scene, assign specific terminology to them, and guide other members' perception of the ongoing activity to achieve a shared mathematical vision.

\section{Coordination of Whiteboard Visualizations and Chat Narratives}

The previous section focused on single actions that refer to some feature of the shared scene for its intelligibility. We argued that such actions involve a part/whole relationship that presumes the availability of a shared visual ground for their mutual intelligibility. In addition to this, such actions are also embedded within broader sequences of actions that establish their relevance. In other words, messages that establish a referential link between narrative and graphical resources routinely respond to practical matters made relevant or projected by prior actions. Thus, such actions are also tied to the context set by the sequentially unfolding discussion.

When the scope of analysis is broadened to sequences of actions that include messages with referential links, one can observe an important affordance of online environments with multiple interaction spaces: Since one can contribute to only one of the interaction spaces at a time, a participant cannot narrate his/her whiteboard actions with simultaneous chat postings, as can be done with talk in a face-to-face setting. However, as we have observed in 137's performance in Excerpts 1 and 7, participants can achieve a similar interactional organization by temporally coordinating their actions in such a way that whiteboard actions can be seen as part of an exposition performed in chat.

For instance, in Excerpt 1, Qwertyuiop's drawing activity was prefaced by his chat posting "I'll draw it". The posting was in response to a recent graphical illustration proposed by 137 . Hence, the pronoun "it" included in the preface was not pointing to an existing drawing or to a prior posting. Instead, it projected a subsequent action to be performed next by the same author. In contrast, prior to Qwertyuiop's actions in Excerpt 1, 137 produced his drawings before he was seen as typing by others. Although the sequence of the chat and whiteboard actions are the opposite in this case (i.e., the referential move was made after the drawing was finished), 137 achieves a similar temporal organization through his use of deictic terms (e.g., "those", "that", "it"), referential arrows, and tokens of similarity such as "like" and "as". Therefore, these instances suggest that, although they can be ordered in different ways, the sequential organization and temporal proximity of actions are consequential for the treatment of a set of drawing actions in relation to a narrative account produced in chat.

In face-to-face settings, locational deictic terms such as "this" and "those" are used to point out contextual elements beyond the lexical content of the uttered statement, and they are often accompanied by cooccurring pointing gestures and body movements displaying the speaker's orientation towards what is being referred to in the vicinity (Hanks, 1992; Goodwin, 2000). As demonstrated by the actual cases of use in the excerpts analyzed above, a similar organization presents an interactional challenge for the participants in an online setting with dual interaction spaces like VMT. However, as participants demonstrated in these excerpts, a functionally comparable interactional organization can be achieved online through the use of available features so that chat messages can be seen as related to shared drawings that are either on display ("those") or in production ("these"). The sequential organization of actions, explicit referencing, and the temporal proximity of actions across both spaces together guide other members' attention so that they can treat such discrete actions as a coherent whole addressed to a particular prior message or to a thread of discussion unfolding at that moment. 
Another important aspect of such achievements from a mathematics education perspective is that it shows us how saming ${ }^{13}$ (Sfard, 2008) among narrative and graphical accounts or realizations can be done as an interactional achievement across dual-interaction spaces. This phenomenon is demonstrated in various episodes such as (a) Qwertyuiop's demonstration of collinear set of lines on the shared diagram in Excerpt 4, and (b) 137's exposition in Excerpt 7, where he showed the geometric implication of his proposal in narrative form by performing a drawing immediately after his chat message. The referential links, the temporal proximity of actions, the awareness indicators for those actions, and the persistent availability of both prior messages and the recently added drawings all work together as a semiotic system that allows group members to make connections among different realizations of the mathematical artifacts that they have co-constructed. Therefore, referential practices across modalities are consequential for the collective achievement of deep understanding of mathematics, which is characterized in mathematics education theory as establishing relationships between different realizations of mathematical ideas encapsulated in graphical, narrative or symbolic forms.

\section{Past and Future Relevancies Implied by Shared Mathematical Artifacts}

The objects on the whiteboard and their visually shared production index a horizon of past and future activities. The indexical terms in many proposals made in the analyzed excerpts (like "hexagonal array", "collinear lines", "rows") not only rely on the availability of the whiteboard objects to propose a relevant activity to pursue next, but also reflexively modify their sense by using linguistic and semantic resources to label or gloss the whiteboard object and its production. This allows actors to orient in particular ways to the whiteboard objects and the procedures of their co-construction-providing a basis for subsequent coordinated joint activity.

This suggests that shared representations are not simply manifestations or externalizations of mental schemas as they are commonly treated in cognitive models of problem-solving processes. Instead, our case studies suggest that shared representations are used as resources to interactionally organize the ways actors participate in collaborative problem-solving activities. As we have seen in this case study, once produced as shared mathematical artifacts, drawings can be mobilized and acted upon as resources for collective reasoning as different members continue to engage with them. Shared meanings of those artifacts are contingently shaped by these engagements, which are performed against the background of a shared visual space including other artifacts and prior chat messages (i.e., against a shared indexical ground). This does not mean that the achievement of shared understanding implies that each member has to develop and maintain mental contents that are isomorphic to each other's, which is often referred as registering shared facts to a "common ground" in psycholinguistics (Clark \& Brennan, 1991). Instead, shared understanding is a practical achievement of participants that is made visible through their reciprocal engagements with shared mathematical artifacts.

The way team members oriented themselves to the shared drawing while they were exploring various properties of the hexagonal array showed that the drawings on the whiteboard have a figurative role in addition to their concrete appearance as illustrations of specific cases. In other words, the particular cases captured by concrete, tangible marks on the whiteboard are routinely used as resources to investigate and talk about the general properties of the mathematical artifacts indexed by them. For example, the particular drawing of the hexagonal pattern in the excerpts studied above was illustrating one particular stage (i.e., $\mathrm{n}=3$ ), yet it was treated in a generic way throughout the whole session as a resource to investigate the properties of the general pattern implied by the regularity/organization embodied in that shared artifact. Noticing of such organizational features motivated the joint development of counting practices, where relevant components of the pattern were first isolated and then systematically counted.

\footnotetext{
${ }^{13}$ Sfard (2008) describes saming as the process of "... assigning one signifier (giving one name) to a number of things previously not considered as being the same" (p. 302).
} 
Another important aspect of the team's achievement of general formulas, which summarize the number of sticks and triangles included in the $\mathrm{n}^{\text {th }}$ case respectively, is the way they transformed a particular way of counting the relevant objects in one of the partitions (i.e., a geometric observation) into an algebraic mode of investigation. For instance, once the team discovered that a particular alignment of sticks that they referred to as "collinear sides" corresponded to triangular numbers, they were able to summarize the sequence of numbers they devised into the algebraic formula $9 n(n+1)-6 n$. The shift to this symbolic mode of engagement, which relied on the presence of shared drawings and prior narratives as resources, allowed the team to progress further in the task of generalizing the pattern of growth by invoking algebraic methods. In other words, the team co-constructed general symbolic formulas for their shared tasks by making coordinated use of multiple realizations (graphical and linguistic) of the mathematical artifact (the hexagonal array) distributed across the dual-interaction spaces.

\section{Conclusion}

Perhaps the most important contribution of online learning environments like VMT to research is that they make the collective mathematical meaning-making process visible to researchers through their logs. This allows us to explore the mechanisms through which participants co-construct mathematical artifacts in graphical, narrative and symbolic forms; and to study how they incrementally achieve a shared understanding of them. Careful analysis of team members' actions helps us identify important affordances (i.e., possibilities and limitations on actions) of digital environments for supporting collaborative discussion of mathematics online.

Our analysis reveals that group members display their reasoning by enacting representational affordances of online environments like VMT. The persistent nature of the contributions and the availability of their production/organization allow other participants to witness the mathematical reasoning embodied in those actions. Group members establish relevancies across graphical, narrative and symbolic realizations of mathematics artifacts by enacting the referential uses of the available system features. Verbal references, highlighting a drawing with different colors, and the explicit referencing feature of the system are used to establish such relationships between contributions. Through referential practices group members:

(a) isolate objects in the shared visual field,

(b) associate them with local terminology stated in chat, and

(c) establish sequential organization among actions performed in chat and whiteboard spaces, which can be expressed in algebraic symbolism.

Finally, this case study also showed us how mathematics terminology comes into being in response to specific communicational needs. Mathematical discourse has a deeply indexical nature; mathematics terminology often encodes certain ways of thinking about mathematical objects. As we have seen in the excerpts above, terminology such as "sides", "collinear set of sides", etc., emerge from the need to talk about and direct others' attention to specific aspects of the task at hand. Such glosses, names or indexicals become meaningful mathematical narrative artifacts through the ways participants enact them by organizing the shared space in particular ways and/or referring to some part of a drawing or a previous chat posting. Once a shared sense of a term is established in interaction, subsequent uses of the term encode certain ways of constructing/grouping/organizing some items and begin to serve as a convenient way to refer to an overall strategy of looking at a problem in a particular way. The term may then lead to a symbolic expression, drawing upon associated practices of computation and manipulation.

In short, mathematical understanding at the group level is achieved through the organization of representational and referential practices. Persistent whiteboard objects and prior chat messages form a shared indexical ground for the group. A new contribution is shaped by the indexical ground (i.e., interpreted in relation to relevant features of the shared visual field and in response to prior actions); it reflexively shapes the indexical ground (i.e., gives further specificity to prior contents) and sets up 
relevant courses of action to be pursued next. Shared mathematical understanding is an observable process, a temporal course of work in the actual indexical detail of its practical actions, rather than a process hidden in the minds of the group members. Deep mathematical understanding can be located in the practices of collective multimodal reasoning displayed by teams of students through the sequential and spatial organization of their actions. Mathematical results are reached through a sequence of discourse interactions that build successively (Stahl, 2011). The discourse moves within the media of graphical constructions, narrative terminology and manipulable symbolisms, allowing progress to be made through visual means, counting skills, encapsulation of knowledge in words, and generalization in symbols.

\section{References}

Barron, B. (2003). When smart groups fail. The Journal of the Learning Sciences. 12(3), 307-359.

CCSSI. (2011). High school -- geometry. In Common Core State Standards Initiative (Ed.), Common core state standards for mathematics. (pp. 74-78)

Çakır, M. P. (2009). The joint organization of visual, narrative, symbolic interactions. In G. Stahl (Ed.), Studying Virtual Math Teams (pp. 99-141). New York, NY: Springer.

Çakır, M. P., Zemel, A., \& Stahl, G. (2009). The joint organization of interaction within a multimodal CSCL medium. International Journal of Computer-Supported Collaborative Learning, 4(2), 115-149.

Carpenter, T. P., \& Lehrer, R. (1999). Teaching and learning mathematics with understanding. In E. Fennema \& T. R. Romberg (Eds.), Mathematics classrooms that promote understanding (pp. 19-32). Mahwah, NJ: Lawrence Erlbaum Associates.

Clark, H. H., \& Brennan, S. (1991). Grounding in communication. In L. B. Resnick, J. M. Levine \& S. D. Teasley (Eds.), Perspectives on Socially Shared Cognition (pp. 127-149). Washington DC: American Psychological Association.

Dillenbourg, P., \& Traum, D. (2006). Sharing Solutions: Persistence and Grounding in Multimodal Collaborative Problem Solving. The Journal of the Learning Sciences, 15(1), 121-151.

Dohn, N. B. (2009). Affordances revisited: Articulating a Merleau-Pontian view. International Journal of Computer-Supported Collaborative Learning, 4(2), 151-170.

Fuks, H., Pimentel, M., \& de Lucena, C. (2006). R-U-Typing-2-Me? Evolving a chat tool to increase understanding in learning activities. International Journal of Computer-Supported Collaborative Learning, 1(1), 117-142.

Garcia, A., \& Jacobs, J. B. (1998). The interactional organization of computer mediated communication in the college classroom. Qualitative Sociology, 21(3), 299-317.

Garfinkel, H. (1967). Studies in Ethnomethodology. Englewood Cliffs, NJ: Prentice Hall.

Garfinkel, H., \& Sacks, H. (1970). On formal structures of practical actions. In J. Mckinney \& E. Tirvakian (Eds.), Theoretical sociology: Perspectives and developments (pp. 337-366). New York, NY: Appleton-CenturyCrofts.

Goodwin, C. (2000). Action and embodiment within situated human interaction. Journal of Pragmatics, 32, 14891522.

Goodwin, C., \& Heritage, J. (1990). Conversation Analysis. Annual Review of Anthropology, 19, 283-307.

Hanks, W. F. (1992). The indexical ground of deictic reference. In A. Duranti \& C. Goodwin (Eds.), Rethinking context: Language as an interactive phenomenon (pp. 43-76). Cambridge, UK: Cambridge University Press.

Hanks, W. F. (2000). Intertexts: Writings on language, utterance, and context. Lanham: Rowman \& Littlefield.

Healy, L., \& Hoyles, C. (1999). Visual and symbolic reasoning in mathematics: Making connections with computers. Mathematical Thinking and Learning, 1(1), 59-84.

Heritage, J. (2002). Oh-prefaced responses to assessments: a method of modifying agreement/disagreement. In C. Ford, B. Fox \& S. Thompson (Eds.), The Language of Turn and Sequence (pp. 196-224). Oxford: Oxford University Press.

Hiebert, J., Carpenter, T. P., Fennema, E., Fuson, K. C., Wearne, D., Murray, H., et al. (1996). Making sense: Teachin and learning mathematics with understanding. Portsmouth NH: Heinemann.

Hiebert, J., \& Wearne, D. (1996). Instruction, Understanding, and Skill in Multidigit Addition and Subtraction. Cognition \& Instruction, 14(3), 251-283.

Jordan, B., \& Henderson, A. (1995). Interaction analysis: Foundations and practice. Journal of the Learning Sciences. 4(1), 39-103. Web: http://1rs.ed.uiuc.edu/students/c-merkel/document4.HTM. 
Kaput, J. (1998). Representations, Inscriptions, Descriptions and Learning: A Kaleidoscope of Windows. Journal of Mathematical Behavior, 17(2), 265-281.

Konold, C. (2007). Designing a data analysis tool for learners. In M. Lovett \& P. Shah (Eds.), Thinking with data: The 33rd annual Carnegie Symposium on cognition (pp. 267-292). Hillside, NJ: Lawrence Erlbaum Associates.

Koschmann, T. (2001). Revisiting the paradigms of instructional technology. In G. Kennedy, M. Keppell, C. McNaught \& T. Petrovic (Eds.), Meeting at the crossroads. Proceedings of the 18th Annual Conference of the Australian Society for Computers in Learning in Tertiary Education (pp. 15-22). Melbourne: University of Melbourne.

Koschmann, T., Stahl, G., \& Zemel, A. (2007). The video analyst's manifesto (or the implications of Garfinkel's policies for the development of a program of video analytic research within the learning sciences). In R. Goldman, R. Pea, B. Barron \& S. Derry (Eds.), Video research in the learning sciences. Mahwah, NJ: Lawrence Erlbaum Associates.

Lagrange, J. B. (2005). Curriculum, classroom practices and tool design in the learning of functions through technology-aided experimental approaches. International Journal for Computers for Mathematical Learning, 10(2), 143-189.

Lenhart, A., Madden, M., Macgill, A. R., \& Smith, A. (2007). Teens and social media. from http://www.pewinternet.org/ /media//Files/Reports/2007/PIP Teens Social Media Final.pdf.pdf

Livingston, E. (1987). Making Sense of Ethnomethodology. London: Routledge and Kegan Paul.

Moss, J., \& Beatty, R. (2006). Knowledge building in mathematics: Supporting collaborative learning in pattern problems. International Journal of Computer-Supported Collaborative Learning, 1(4), 441-465.

Mühlpfordt, M., \& Stahl, G. (2007). The integration of synchronous communication across dual interaction spaces. Paper presented at the International Conference of CSCL: Of mice, minds, and society (CSCL 2007). New Brunswick, NJ. Web: http://GerryStahl.net/vmtwiki/martin.pdf.

NCTM. (2000). Principles and standards for school mathematics. Reston, VA: National Council of Teachers of Mathematics.

Olive, J. (1998). Opportunities to explore and integrate mathematics with the geometer's sketchpad. In R. Lehrer \& D. Chazan (Eds.), Designing learning environments for developing understanding of geometry and space. (pp. 395-418). Mahwah, NJ: Lawrence Erlbaum.

O'Neil, J., \& Martin, D. (2003). Text chat in action. Paper presented at the The International ACM SIGGROUP conference on Supporting group work, Sanibel Island, Florida, USA.

Sacks, H. (1962/1995). Lectures on Conversation. Oxford, UK: Blackwell.

Sarmiento, J., \& Stahl, G. (2008). Extending the joint problem space: Time and sequence as essential features of knowledge building. Paper presented at the International Conference of the Learning Sciences (ICLS 2008). Utrecht, Netherlands.

Schegloff, E. A., \& Sacks, H. (1973). Opening up closings. Semiotica, 7, 289-327.

Sfard, A. (2002). There is more to discourse than meets the ears: Looking at thinking as communicating to learn more about mathematical learning. In C. Kieran, E. Forman \& A. Sfard (Eds.), Learning discourse: Discursive approaches to research in mathematics (pp. 13-57). Dordrecht, Netherlands: Kluwer.

Sfard, A. (2008). Thinking as communicating: Human development, the growth of discourses, and mathematizing. New York, NY: Cambridge University Press.

Skemp, R. R. (1976). Relational understanding and instrumental understanding. Mathematics Teaching, 77, $20-26$.

Stahl, G. (2006). Group Cognition: Computer Support for Building Collaborative Knowledge. Cambridge, MA: MIT Press.

Stahl, G. (Ed.). (2009). Studying virtual math teams. New York, NY: Springer.

Stahl, G. (2011). How a virtual math team structured its problem solving. Paper presented at the international conference on Computer-Supported Collaborative Learning (CSCL 2011). Hong Kong, China. Proceedings pp. 256-263.

Stahl, G., Koschmann, T., \& Suthers, D. D. (2006). Computer-Supported Collaborative Learning. In R. K. Sawyer (Ed.), The Cambridge Handbook of the Learning Sciences (pp. 409-426). New York: Cambridge University Press.

Stahl, G., Zhou, N., Çakır, M. P., \& Sarmiento-Klapper, J. W. (2011). Seeing what we mean: Co-experiencing a shared virtual world. Paper presented at the international conference on Computer Support for Collaborative Learning (CSCL 2011). Hong Kong, China. Proceedings pp. 534-541.

Suthers, D., Connelly, J., Lesgold, A., Paolucci, M., Toth, E., Toth, J., et al. (2001). Representational and Advisory Guidance for Students Learning Scientific Inquiry. In K. D. Forbus \& P. J. Feltovich (Eds.), Smart 
Machines in Education: The Coming Revolution in Educational Technology (pp. 7-35). Menlo Park: AAAI Press.

Suthers, D. D. (2006). Technology affordances for intersubective meaning making: A research agenda for CSCL. International Journal of Computer-Supported Collaborative Learning (ijCSCL), 1(3), 315-337.

ten Have, P. (1999). Doing conversation analysis, A practical guide. Thousand Oaks, CA: Sage Publications.

Watson, A., \& Mason, J. (2005). Mathematics as a constructive activity: Learners generating examples. Mahwah, NJ: Lawrence Erlbaum Associates.

Zemel, A., \& Çakır, M. P. (2009). Reading's Work in VMT. In Stahl, G. (Ed.), Studying Virtual Math Teams. (pp. 261-276). New York, NY: Springer. 